\title{
Response Transformation and Receptive-Field Synthesis in the Lemniscal Trigeminothalamic Circuit
}

\author{
Brandon S. Minnery, Randy M. Bruno, and Daniel J. Simons \\ Department of Neurobiology, University of Pittsburgh, Pittsburgh, Pennsylvania 15261 \\ Submitted 5 February 2003; accepted in final form 25 April 2003
}

\begin{abstract}
Minnery, Brandon S., Randy M. Bruno, and Daniel J. Simons. Response transformation and receptive-field synthesis in the lemniscal trigeminothalamic circuit. J Neurophysiol 90: 1556-1570, 2003. First published April 30, 2003; 10.1152/jn.00111.2003. To understand how the lemniscal trigeminothalamic circuit (PrV $\rightarrow$ VPM) of the rodent whisker-to-barrel pathway transforms afferent signals, we applied ramp-and-hold deflections to individual whiskers of lightly narcotized rats while recording the extracellular responses of neurons in either the ventroposterior medial (VPM) thalamic nucleus or in brain stem nucleus principalis (PrV). In PrV, only those neurons antidromically determined to project to VPM were selected for recording. We found that VPM neurons exhibited smaller response magnitudes and greater spontaneous firing rates than those of their PrV inputs, but that both populations were similarly well tuned for stimulus direction. In addition, fewer VPM (74\%) than PrV neurons (93\%) responded with sustained, or tonic, discharges during the plateau phase of the stimulus. Neurons in both populations responded most robustly to deflections of a single, "principal whisker" (PW), and the majority of cells in both PrV (90\%) and VPM (73\%) also responded to deflections of at least one adjacent whisker (AW). AW responses in both nuclei occurred on average at longer latencies and were more temporally dispersed than PW responses. Lateral inhibition, as evidenced by AW-evoked activity suppression, was rare in PrV but prevalent in VPM. In both nuclei, however, suppression was weak, with AW responses being on average excitatory. Our results suggest that the receptive-field structures and response properties of individual VPM neurons can be explained in large part by input from one or a small number of PrV neurons, but that intrathalamic mechanisms act to further transform the afferent signal.
\end{abstract}

\section{N T R O D U C T I O N}

In the rodent whisker-to-barrel system, sensory information from the large facial whiskers ascends from the periphery to the cortex by two major routes, a lemniscal pathway and a parallel, or "paralemniscal," pathway. Within the lemniscal route, afferent signals are processed at the subcortical level by a trigeminothalamic circuit consisting prominently of feedforward projections from brain stem nucleus principalis (PrV) to the ventroposterior medial (VPM) nucleus of the thalamus. In VPM, stereotyped modules of neurons termed "barreloids" (Van der Loos 1976) replicate in a one-to-one fashion the pattern of mystacial whiskers on the contralateral face. Each barreloid receives its major afferent drive from a corresponding "barrelette" (Ma 1991) in PrV and in turn sends its axons to the somatotopically matched "barrel" (Woolsey and Van der Loos

Address for reprint requests: D. J. Simons, E 1440, Biomedical Science Tower, 200 Lothrop Street, University of Pittsburgh, Pittsburgh, PA 15261 (E-mail: cortex@pitt.edu).
1970) in layer IV of primary somatosensory cortex (SI). Understanding the input-output transformations performed by the $\mathrm{PrV} \rightarrow$ VPM circuit thus represents an essential step toward understanding the nature of signal processing within afferent subcortical pathways.

Unlike the visual system, where detailed anatomical (Hamos et al. 1987; Wilson 1989) and cross-correlation studies (Mastronarde 1987, 1992; Usrey et al. 1999) have contributed to our knowledge of retinogeniculate circuitry, far less is known regarding information processing in the PrV $\rightarrow$ VPM pathway. Recently however, a number of elegant anatomical studies have begun to illuminate the detailed organization of barreloid circuitry in the rat, including the dendroarchitecture of VPM neurons (Chiaia et al. 1991b; Ohara and Havton 1994; Varga et al. 2002), the termination patterns of their afferent inputs (Chiaia et al. 1991a; Pierret et al. 2000; Varga et al. 2002; Veinante and Deschênes 1999; Veinante et al. 2000; Williams et al. 1994), and the anatomical arrangement of inhibitory feedback projections (Crabtree et al. 1998; Desîlets-Roy et al. 2002; Pinault and Deschênes 1998a,b; Varga et al. 2002). VPM neurons have also been shown to receive extensive feedback excitation from the deep layers of barrel cortex (reviewed in Deschênes et al. 1998) as well as feedforward neuromodulatory inputs from the brain stem (Bennett-Clarke et al. 1999; Simpson et al. 1997).

The findings put forth in the preceding studies have challenged longstanding perceptions of the thalamus as a passive relay station and, together with recent physiological studies (Ahissar et al. 2000; Castro-Alamancos 2002a,b; Fanselow and Nicolelis 1999; Fanselow et al. 2001; Sosnik et al. 2001), have raised new questions as to the dynamic nature of information transfer at the trigeminothalamic synapse. For example, recordings from both trigeminal pathways by Ahissar and colleagues (Ahissar et al. 2000; Sosnik et al. 2001) have demonstrated that significant temporal processing of afferent information occurs within the paralemniscal circuit, whereas the lemniscal circuit appears to be primarily involved in processing the spatial details of tactile stimuli. Indeed, we recently found that VPMprojecting PrV neurons have spatially focused receptive fields that, although multiwhisker, are dominated by a single vibrissa (Minnery and Simons 2002; see also Shipley 1974).

In the present study, we compare the stimulus-evoked responses of antidromically identified, VPM-projecting PrV neurons to those of VPM neurons to further characterize the role

\footnotetext{
The costs of publication of this article were defrayed in part by the payment of page charges. The article must therefore be hereby marked "advertisement" in accordance with 18 U.S.C. Section 1734 solely to indicate this fact.
} 
of the lemniscal trigeminothalamic circuit in transforming afferent signals. Our data indicate that the $\operatorname{PrV} \rightarrow$ VPM circuit preserves the spatial features and directional characteristics of tactile inputs as well as important temporal properties of stimulus-evoked responses. However, we find that VPM neurons respond overall less robustly to ramp-and-hold whisker deflections than do PrV cells, particularly during the plateau phase of the stimulus when feedback inhibition from the thalamic reticular nucleus is pronounced (Hartings et al. 2000). Moreover, the relative sizes of responses to stimulus offset compared with onset are elevated in VPM versus PrV neurons, consistent with previous studies (Kyriazi et al. 1994; see also Sherman 2001 for review), suggesting a role for postinhibitory rebound mechanisms within the thalamic circuit.

\section{METHODS}

\section{Surgical preparation}

$\operatorname{PrV}$ and VPM data were obtained from two separate groups of female Sprague-Dawley rats weighing $250-300 \mathrm{~g}$. The procedures for preparing animals for recording were previously described in detail (Minnery and Simons 2003; Simons and Carvell 1989). Briefly, during an initial surgery conducted under halothane anesthesia, a tracheal cannula was inserted by means of a tracheotomy, catheters were inserted into the right jugular vein and left femoral artery, and a steel post was affixed to the skull for the purpose of stabilizing the head during recording sessions. For those animals prepared for PrV recordings, one craniectomy was made in a region overlying the rostral half of the left brain stem trigeminal complex. A second craniectomy was then made over the right ventrobasal thalamus (VB) to allow access to contralateral VPM with an antridromic-stimulating electrode. For those animals prepared for VPM recordings, a craniectomy was made overlying the right VB. Halothane was discontinued after surgery, and animals were transferred to a vibration-isolation table and switched to a mechanical ventilator. Throughout recording sessions, rats were maintained in a sedated and lightly narcotized state by continuous intravenous delivery of a synthetic narcotic, fentanyl citrate (Sublimaze, Jansen Pharmaceuticals; $10 \mu \mathrm{g} \mathrm{kg}^{-1} \mathrm{~h}^{-1}$ ). To prevent spontaneous movements of the whiskers, which would preclude use of our electromechanical stimulators (see following text), rats were immobilized by intravenous infusion of pancuronium bromide $\left(1.6 \mathrm{mg} \mathrm{kg}^{-1} \mathrm{~h}^{-1}\right)$. An animal's physiological state was continuously assessed by a computer that monitored heart rate, femoral arterial blood pressure, tracheal airway pressure waveform, and, in the case of VPM recordings, electroencephalogram (EEG). Core body temperature was maintained at $37^{\circ} \mathrm{C}$ by means of a servo-controlled heating blanket.

\section{Electrophysiological recordings}

The PrV data reported here as well as related recording procedures were recently described (Minnery and Simons 2003). Extracellular single-unit recordings of VPM-projecting neurons in the left PrV were accomplished using either stainless steel or platinum-tungsten microelectrodes (500 k $\Omega-3 \mathrm{M} \Omega$ ), and data are reported only for units that were antidromically determined to project to VPM (see Minnery and Simons 2003 for additional details). VPM data were also taken from a recent study (Bruno and Simons 2002). Extracellular single-unit recordings were obtained by 5 - to $12-\mathrm{M} \Omega$ quartz-insulated platinumtungsten microelectrodes. For both studies, data in the form of spike waveforms and/or digitized spike times were collected using PCbased acquisition hardware from National Instruments (Austin, TX) in conjunction with custom-written LabView software (National Instruments). Details are provided in the original studies. Analog signals were digitized at $32 \mathrm{kHz}$ by a $1-\mathrm{GHz}$ PC equipped with a PCI-MIO$16 \mathrm{E}-1$ board (National Instruments).

Because of the nature of the original study, VPM neurons were recorded principally while in the tonic firing mode. A cell was qualitatively determined to be in tonic firing mode if its spontaneous activity did not contain substantial spike bursts separated by long periods of quiescence (see Sherman 2001 for review). The selective recording of VPM cells in tonic mode may explain subtle discrepancies in response parameters between the present data and previous studies from our laboratory (Hartings et al. 2000; Simons and Carvell 1989). For instance, both spontaneous and plateau firing rates are greater during tonic firing mode, when cell membranes are relatively depolarized and neurons respond in a linear fashion to stimuli (see Sherman 2001; Fig. 2). However, when comparing our current VPM data to that of earlier reports (cited above), we found that differences were not so robust as to alter the essential conclusions of this study.

\section{Whisker stimulation and receptive-field classification}

A handheld probe was initially used to identify the whisker most effective at evoking activity in an isolated cell [i.e., the principal whisker (PW)]. A piezoelectric mechanical stimulator (Simons 1983) was then attached to the PW approximately $10 \mathrm{~mm}$ from the skin surface. Ramp-and-hold deflections were applied 10 times in each of 8 directions differing in $45^{\circ}$ increments, for a total of 80 trials. For the majority of PW recordings in VPM (46 of 77 cells), this procedure was repeated twice for a total of 160 trials. In all other cases 80 trials were conducted. Interstimulus intervals of $\geq 2 \mathrm{~ms}$ separated the offset of one whisker deflection from the onset of the next. At these low stimulus frequencies, it is unlikely that frequency-dependent response adaptation influenced our results for either PrV (Sosnik et al. 2001; Minnery, unpublished data) or VPM neurons (Castro-Alamancos 2002b; Hartings and Simons 1998; Sosnik et al. 2001).

Ramp-and-hold stimulus waveforms were similar to those employed in previous studies in our laboratory and were generated by D/A converters. Whisker deflections were $1 \mathrm{~mm}$ in amplitude, with onset/offset velocities of $125 \mathrm{~mm} / \mathrm{s}$ and a plateau duration of $200 \mathrm{~ms}$. The angle of deflection was varied in a pseudorandom order throughout the stimulus presentation. This procedure was then repeated for several whiskers adjacent to the PW. When possible, we recorded the responses of the rostral, caudal, ventral, and dorsal adjacent whiskers (AWs). For two PrV cells whose PW consisted of the $\Delta$ "straddler" whisker situated between rows D and E, only the dorsal AW $(\gamma)$ response is described here. None of the VPM cells in our sample had a straddler whisker as the PW. With the exception of the two aforementioned PrV cells, no straddler whiskers were included as AWs for either the PrV or VPM populations.

To facilitate comparison with our recent study of $\operatorname{PrV}$ receptive fields (Minnery and Simons 2003), a cell's PW in the present study was defined as the whisker evoking the largest response to stimulus onset (averaged over all angles). By definition, therefore, AW responses for a given cell in the present study are weaker than those of the PW. In contrast, earlier studies of VPM neurons in our laboratory (Simons and Carvell 1989) employed criteria that allowed for the occasional classification of AWs whose evoked responses were slightly more robust than those of PWs. The classification criterion used in the current study thus provides a more conservative estimate of relative receptive-field size.

\section{Response detection/quantification}

Spike times were measured with $100-\mu$ s resolution and were compiled into peristimulus time histograms (PSTHs) having 1-ms bin width. For each deflection angle, response magnitudes for stimulus onset (ON) and offset (OFF) were defined as the total number of spikes occurring within a 20-ms response window. ON and OFF windows were positioned so that the onset of the window coincided with the earliest 
onset of the response as determined by the population PSTHs for each nucleus. Plateau responses were defined as the number of spikes occurring over the middle $100 \mathrm{~ms}$ of the plateau epoch. Spontaneous (i.e., background) activity measurements for a given cell were derived from a corresponding AW data file for that cell and were calculated over a $100-\mathrm{ms}$ period of prestimulus activity. We calculated spontaneous activity from AW rather than PW data because prestimulus firing rates for PW files were often elevated simply by attachment of the stimulator to the PW. Because AWs were typically much less responsive than PWs, this spurious enhancement of prestimulus activity was seldom observed for AW data. We did not subtract spontaneous/background activity when reporting response magnitudes because it was not clear that the number of spikes within a response window could be accurately described as the linear sum of stimulusevoked and background activities. However, we indicate throughout the text where differences in background activity levels between PrV and VPM populations might influence interpretations of numerical data.

We tested for the presence of statistically significant excitatory responses within a specific response window by comparing firing rates at the maximally responsive angle to prestimulus firing rates estimated over a $100-\mathrm{ms}$ period ( $\alpha=0.05$; one-tailed $t$-test). The maximally responsive angle was chosen to avoid the misclassification of highly directionally tuned cells as unresponsive. The same criterion was employed to assess activity suppression within a given response window, with the exception being that the minimally responsive angle was selected for analysis. When testing for suppression evoked by stimulus onset, we used a longer ON window $(40 \mathrm{~ms})$ to accommodate the effects of long-latency feedback inhibition. In the case of VPM responses, the start of this window was offset by $4 \mathrm{~ms}$ from the start of the standard on window to accommodate the exclusively feedback nature of inhibition in rat VPM (Barbaresi et al. 1986; Harris and Hendrickson 1987). We did not examine stimulus offset-evoked suppression in this study. Given that activity suppression is difficult to infer for cells having little or no spontaneous activity, we excluded from analysis those cells having prestimulus firing rates $<6 \mathrm{~Hz}$. The 6-Hz cutoff value was empirically determined through inspection of individual PSTHs, which suggested that, because of statistical artifact, AWs were more likely to be misidentified as suppressive when spontaneous firing rates were low. However, even when cells with firing rates below $6 \mathrm{~Hz}$ were included in our analyses (data not shown), our essential findings regarding the prevalence of AWevoked suppression in PrV and VPM (see REsults, Fig. 6) did not change. In keeping with previous studies (Hartings et al. 2000; Lichtenstein et al. 1990; Simons and Carvell 1989), we used a slightly more stringent statistical criterion $(\alpha=0.025)$ when testing for the presence of plateau responses (i.e., when classifying cells as "tonic" vs. "phasic").

\section{Temporal characterization of responses}

The temporal characteristics of stimulus-evoked responses were quantified according to several measures. 1) $T_{50 \%}$. As a measure of the temporal distribution of a neuron's response, we computed the time to the 50th percentile spike $\left(\mathrm{T}_{50 \%}\right)$ within a given response window relative to the start of the window (Pinto et al. 2000). For each cell, all deflection angles were included in the computation of $\mathrm{T}_{50 \%}$. We also computed population $\mathrm{T}_{50 \%}$ values based on the all-angles population PSTHs for each nucleus. 2) Latency to first spike. The latency to occurrence of the first spike within a given response window relative to the start of the window was determined for each trial (i.e., each deflection) for which at least one spike was present. These latencies were then averaged to yield a mean latency per whisker per neuron. We interpret this mean latency to represent the earliest onset time of the suprathreshold response. The onset of the response window was used as a reference point rather than the onset of the stimulus, which occurred several milliseconds earlier, to minimize the potential con- tribution of spontaneously occurring spikes to our latency measurements. 3) Latency to response onset. To determine the absolute latency of a neuron's response relative to the onset of the stimulus, we compared spike counts within each 1-ms bin of the ON window to spike counts measured over 100 bins of prestimulus activity. The latency to response onset was defined as the first of two consecutive bins within the oN window for which spike counts significantly exceeded prestimulus levels $(P<0.05$ per bin). Prestimulus activity was modeled as a Poisson distribution. [Note: the significance level employed here, $\alpha=0.05$, is slightly less strict than that used in an earlier study of PrV response latencies by our laboratory (Minnery and Simons 2003). This explains the small discrepancy between the PrV latencies reported in the current vs. the earlier study. The significance level was relaxed in the present study to allow for the incorporation of more VPM AW responses, whose overall response magnitudes were less robust relative to prestimulus firing rates.] To examine whether the criterion for two consecutive bins might have been too conservative, we repeated our analysis with the requirement that only one bin exceed prestimulus levels. This resulted in the detection of many more "responses" that were readily identified as false positives because of their unrealistically short latencies. We therefore adhered to the two-bin criterion used by Minnery and Simons (2003).

\section{Histology}

At the conclusion of experiments, marker lesions were made by passing current ( $7 \mu \mathrm{A}$ for $7 \mathrm{~s}$ ) through the recording electrode and, in the case of PrV recordings, through the thalamic stimulating electrode. Rats were then deeply anesthetized with sodium pentobarbital (Nembutal) and perfused transcardially. Brains were frozen, serially sectioned $(60 \mu \mathrm{m})$, processed for cytochrome oxidase (CO) histochemistry, and counterstained with thionin. Tissue was visually inspected to confirm the presence of electrode tracks in the appropriate nuclei. No consistent attempt was made to resolve recording locations to the level of individual barreloids/barrelettes. Recording sites were histologically confirmed for all of 77 VPM neurons and 69 of $72 \mathrm{PrV}$ neurons. For three cells in our PrV data set, recording location was determined based solely on physiological criteria.

\section{RE S ULTS}

Here we compare the response properties of antidromically identified VPM-projecting PrV neurons $(n=72 ; 16$ rats) with those of VPM neurons ( $n=77 ; 33$ rats) recorded in a separate group of animals under similar experimental and stimulus conditions. Both the PrV and VPM data reported here were previously described (PrV: Minnery and Simons 2003; VPM: Bruno and Simons 2002). For each neuron in both PrV and VPM populations, ramp-and-hold stimuli were applied to the principal excitatory whisker (PW) and up to 4 adjacent whiskers (AWs) in succession. During recordings, cells were targeted whose receptive fields consisted of the large, caudal whiskers of the ventral rows (arcs $1-4$, rows $\mathrm{C}-\mathrm{E}$ ), given that these whiskers were most readily accessed with our stimulator.

\section{Spontaneous and stimulus-evoked activities}

Figure 1 depicts the population responses of PrV and VPM neurons to ramp-and-hold deflections of the PW. Responses are displayed as PSTHs and include data from all 8 deflection angles. Several key differences as well as similarities are captured in the response profiles shown in Fig. 1. Most notice- 
PrV

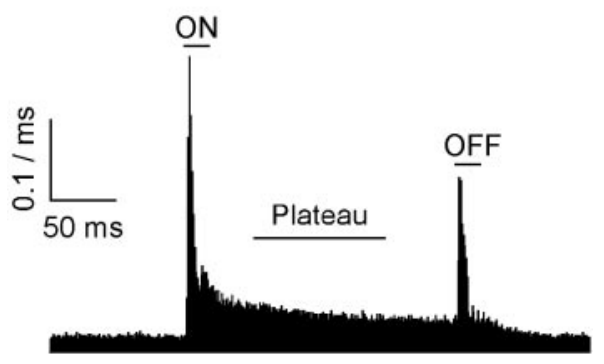

VPM

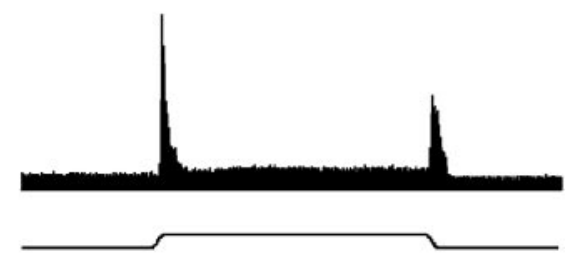

VPM - PRV

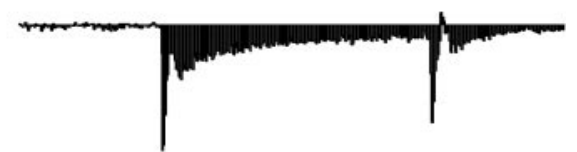

FIG. 1. Brain stem nucleus principalis (PrV) and ventroposterior medial thalamic nucleus (VPM) population responses. Top: peristimulus time histogram (PSTH) showing PrV population response to ramp-and-hold deflections of principal whisker (PW) $(n=72$ cells; 5,760 deflections). Units on vertical scale bar are spike probability/ms. Labeled lines over PSTH indicate three response windows most frequently examined in this study. Middle: VPM population PSTH ( $n=77$ cells; 9,760 deflections). Line under PSTH depicts stimulus waveform. Bottom: population response differential (VPM - PrV). PrV and VPM PSTHs were temporally aligned before subtraction.

ably, both populations exhibited substantial spontaneous (i.e., prestimulus) activity, both responded transiently to stimulus onset (ON) and offset (OFF), and both displayed sustained discharges above prestimulus levels throughout the plateau phase of the stimulus, albeit at lower rates than during the ON and OFF phases. Also readily apparent, however, are substantial differences in response magnitude and time course between $\operatorname{PrV}$ and VPM (Fig. 1, bottom). These differences encompassed all phases of the stimulus-evoked response, with VPM neurons having weaker responses on average than those of their presynaptic counterparts in PrV.

Figure 2 summarizes for each population the mean response magnitudes at both the maximally and minimally effective angles, as well as the mean responses averaged over all 8 angles (see also Table 1). As suggested by the PSTHs in Fig. 1, VPM cells responded to PW deflections with significantly lower firing rates than $\operatorname{PrV}$ cells for all response windows (all values of $P<0.05$; Mann-Whitney $U$ test). In contrast, we found that spontaneous (i.e., background) activity levels were greater in VPM than in PrV $(15.62 \pm 9.21$ vs. $11.90 \pm 12.70 \mathrm{~Hz}: P<0.0005$; MannWhitney $U$ test). The population data thus indicate an overall decrease in signal magnitude relative to background within the trigeminothalamic pathway. The same result was also found at the level of individual neurons. For each cell, we calculated the ratio of spontaneous activity to on re- sponse magnitude (spontaneous activity was placed in the numerator because values were occasionally very small). Separate ratios were computed for on responses at the maximally responsive angle (best angle) and for the average ON response over all angles (see Table 1). In both cases, VPM cells were found to have greater spontaneous:on ratios, or smaller signal:background ratios, than $\operatorname{PrV}$ cells (both values of $P<0.0005$; Mann-Whitney $U$ test).

A previous study from our laboratory (Kyriazi et al. 1994)
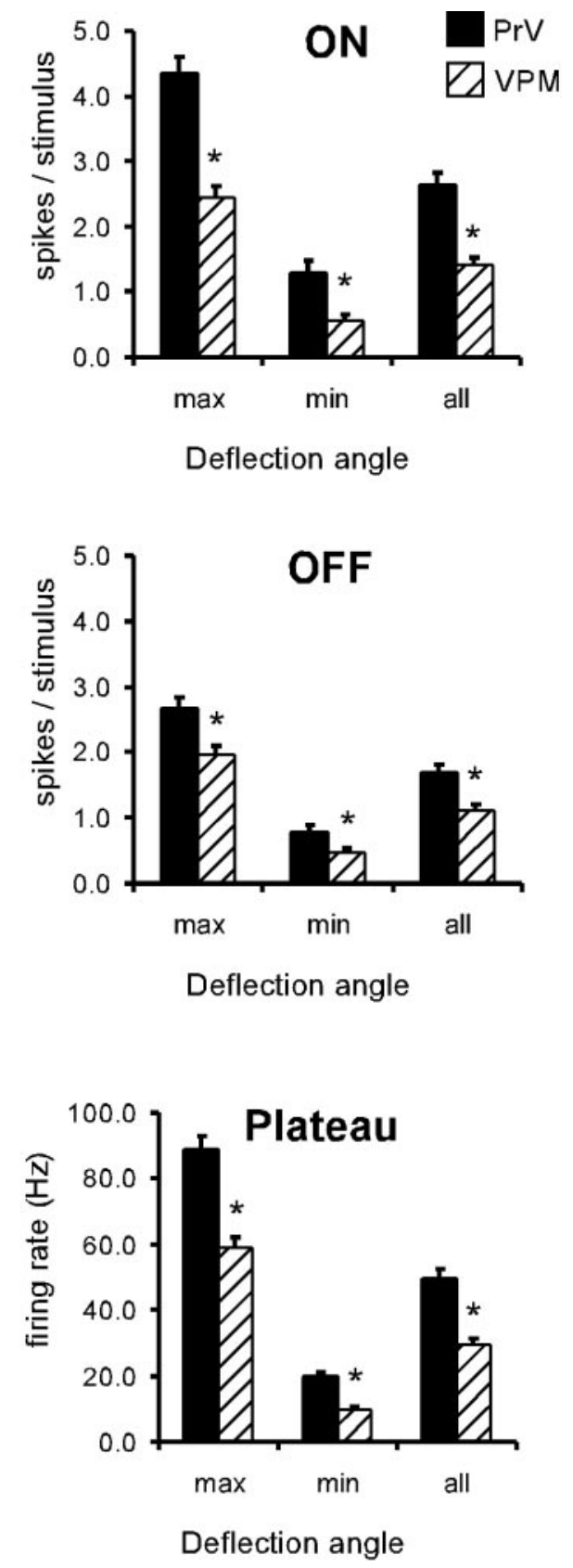

FIG. 2. Comparison of stimulus-evoked activity in PrV and VPM. All values are population means $+1 \mathrm{SE}$. OFF and ON responses were computed over 20-ms windows; plateau responses represent mean firing rate over middle 100-ms period of stimulus plateau (see Fig. 1). "Max" and "min": maximally and minimally responsive deflection angles. "All": average response for all deflection angles $(n=8)$. All data are for PW deflections only (PrV: $n=72$; VPM: $n=77)$. *Statistically significant difference between PrV and VPM means $(P<0.05$; Mann-Whitney $U$ test $)$. 
TABLE 1. Stimulus-evoked and spontaneous activities of $\operatorname{PrV}$ and VPM neurons

\begin{tabular}{|c|c|c|}
\hline Activity & PrV & VPM \\
\hline \multicolumn{3}{|l|}{ ON§ } \\
\hline $\max$ & $4.33 \pm 2.21$ & $2.45 \pm 1.46^{*}$ \\
\hline $\min$ & $1.31 \pm 1.35$ & $0.56 \pm 0.73^{*}$ \\
\hline all & $2.65 \pm 1.57$ & $1.41 \pm 0.95^{*}$ \\
\hline \multicolumn{3}{|l|}{$\mathrm{OFF} \S$} \\
\hline $\max$ & $2.68 \pm 1.32$ & $1.97 \pm 1.19^{*}$ \\
\hline $\min$ & $0.79 \pm 0.83$ & $0.47 \pm 0.66^{*}$ \\
\hline all & $1.70 \pm 1.00$ & $1.11 \pm 0.82^{*}$ \\
\hline $\mathrm{OFF}_{\max } / \mathrm{ON}_{\max }$ & $0.65 \pm 0.21$ & $0.84 \pm 0.35^{*}$ \\
\hline $\mathrm{OFF}_{\text {all }} / \mathrm{ON}_{\text {all }}$ & $0.68 \pm 0.24$ & $0.81 \pm 0.35^{*}$ \\
\hline \multicolumn{3}{|l|}{ Plateau $\dagger$} \\
\hline $\max$ & $8.92 \pm 4.42$ & $5.89 \pm 2.89^{*}$ \\
\hline $\min$ & $1.99 \pm 2.43$ & $0.96 \pm 1.04 *$ \\
\hline all & $5.00 \pm 2.68$ & $2.95 \pm 1.72 *$ \\
\hline Spontaneous $\ddagger$ & $11.90 \pm 12.70$ & $15.62 \pm 9.21^{*}$ \\
\hline Spont./ON $\max$ & $0.07 \pm 0.08$ & $0.15 \pm 0.10 *$ \\
\hline Spont./ON all & $0.12 \pm 0.14$ & $0.26 \pm 0.17 *$ \\
\hline
\end{tabular}

All values are mean $( \pm \mathrm{SD})$ spikes per stimulus unless otherwise indicated. $\operatorname{PrV}$, nucleus principalis; VPM, ventroposterior medial nucleus of the thalamus; ON and OFF, responses to stimulus onset and offset; "max" and "min," maximally and minimally responsive deflection angles; "all," average over all 8 angles. Note: only responses to principal whisker deflections are presented here (PrV: $n=72$ cells; VPM: $n=77$ cells). $\S$ ON and OFF windows are $20 \mathrm{~ms}$ in duration (see Fig. 1). † Plateau responses are calculated over a $100-\mathrm{ms}$ window (see Fig. 1). Only "tonic" plateau responses are included in the calculation of population means (see METHODS). $\ddagger$ Spontaneous firing rates $(\mathrm{Hz})$ for a given cell are calculated from 100-ms epochs of prestimulus data (PrV: $n=72$ cells; VPM: $n=60$ cells). * Difference between PrV and VPM is significant $(P<0.05$; Mann-Whitney $U$ test $)$.

suggested that low-threshold calcium currents $\left(I_{T}\right)$, which are known to exist in VPM neurons, may act to enhance VPM OFF response magnitudes through the generation of postinhibitory spike bursts (see also Sherman 2001). It was postulated by Kyriazi and colleagues that this enhancement should be reflected as an increase in the relative magnitude of OFF versus ON responses in the VPM population compared with $\operatorname{PrV}$, with response magnitude defined as in the present study (i.e., total spike count within the response window). As shown in Fig. 2, this was indeed the case for mean ON and OFF responses. VPM OFF response magnitudes, although smaller than those of their $\mathrm{PrV}$ inputs, were relatively larger compared to VPM oN responses.

To investigate this issue further, we computed for each neuron an OFF:ON response ratio for both the best-angle responses $\left(\mathrm{OFF}_{\max } / \mathrm{ON}_{\max }\right)$ and the average responses over all angles $\left(\mathrm{OFF}_{\text {all }} / \mathrm{ON}_{\text {all }}\right)$ (see Table 1). Under both conditions, the average OFF:ON ratio for VPM neurons was greater than that for PrV neurons (both values of $P<0.01$; Mann-Whitney $U$ test). These differences were found to persist after adjustment of responses for background activity, and therefore cannot be explained by higher spontaneous firing rates of VPM cells (data not shown). Also consistent with the observations of Kyriazi and colleagues, OFF responses in VPM were more temporally dispersed than on responses as measured by $\mathrm{T}_{50 \%}$ (see METHODS; $P<0.0005$; paired $t$-test). In $\operatorname{PrV}$, on the other hand, ON and OFF responses had similar $\mathrm{T}_{50 \%}$ values $(P=0.71$; see Table 3 for mean $\mathrm{T}_{50 \%}$ values and Fig. 8 for higherresolution PSTHs). Thus differences in the relative magnitudes and temporal structures of ofF versus on responses appear to be established principally within VPM.

\section{Directional tuning}

Consistent with previous reports, we found that the majority of cells in both PrV and VPM exhibited some degree of sensitivity to the direction of whisker deflection. Although this property was frequently observed for all phases of a cell's response (i.e., ON, OFF, plateau), here we analyzed only the directional tuning of on responses (see Minnery and Simons 2003 and Simons and Carvell 1989 for additional discussions of directional tuning in PrV and VPM, respectively). We employed several analyses to quantify directional tuning, the first two of which used normalization procedures to enable comparison between cells and populations whose response magnitudes often differed substantially. First, we computed normalized, rotated polar plots for each population (Fig. 3A). For each cell, we normalized the on response magnitude at all angles with respect to the response at the maximally responsive angle. Individual polar plots were then rotated so that maximally responsive angles were aligned in a common direction, and an average response at each angle was then calculated based on the entire population. As is evident in Fig. 3A, both PrV and VPM were well tuned for stimulus direction, and the degree of directional tuning was strikingly similar between both populations.

As a second measure of directional tuning, we calculated for each cell a tuning ratio, defined as the average on response (all angles) divided by the on response at the best angle. In accordance with Fig. $3 A$, Fig. $3 B$ shows that the distributions of tuning ratios for both populations were similar. Mean tuning ratios ( \pm SD) for PrV and VPM were, respectively, $0.58 \pm$ 0.13 and $0.61 \pm 0.15$. Although there was a slight tendency toward lower tuning ratios in PrV than in VPM, indicating greater directional tuning within the PrV population, this difference was not significant $(P=0.13$; Mann-Whitney $U$ test $)$.

We also computed for each cell a tuning index (Fig. 3C), whereby a cell was classified according to the number of deflection angles $(0-7)$ that elicited responses that were statistically smaller than the response at the best angle $(P<0.05$; one-tailed $t$-test). Accordingly, an index of 7 indicates the highest degree of tuning, whereas 0 indicates a nontuned cell. Again the results reveal a strong tendency toward directional selectivity within both populations, with VPM cells expressing slightly less directional tuning than PrV cells. For both PrV and VPM, the majority of neurons were classified in the 4 highest tuning categories (indices $4-7 ; \operatorname{PrV}=69 \%, \mathrm{VPM}=60 \%$ ). The percentage of cells having indices $4-7$ was found not to differ significantly between populations $\left(P=0.22 ; \chi^{2}\right.$ test $)$. Taken together, the above 3 analyses indicate that the directional properties of a stimulus, as encoded in neuronal firing patterns, are for the most part faithfully preserved across the trigeminothalamic synapse.

\section{Adaptive properties}

Previous studies have suggested that the expression of plateau responses within the VPM population is regulated by intrinsic thalamic circuitry, with inhibitory feedback from the thalamic reticular nucleus (Rt) functioning to suppress the effects of sustained, or tonic, inputs arriving from $\operatorname{PrV}$ (Gottschaldt et al. 1983; Hartings and Simons 2000; Lee et al. 1994a,b). In the present study, we explored this hypothesis by 
A

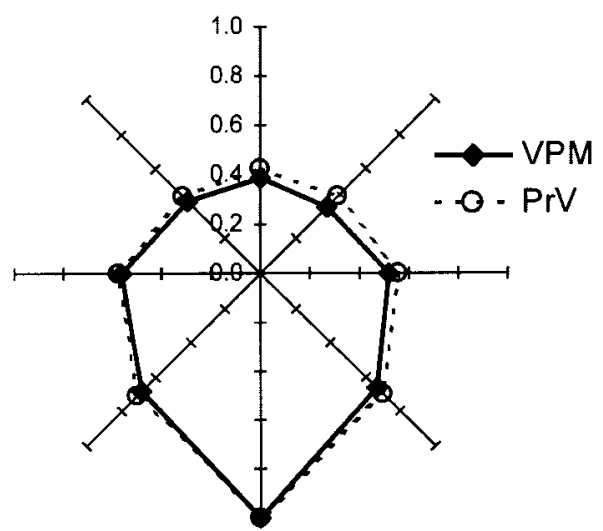

B

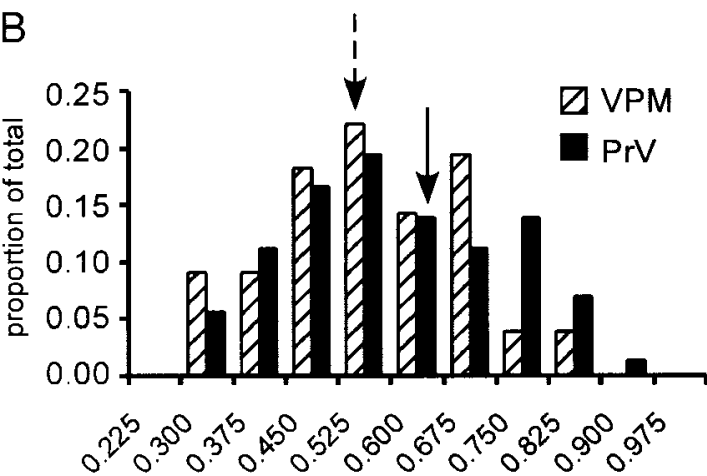

tuning ratio

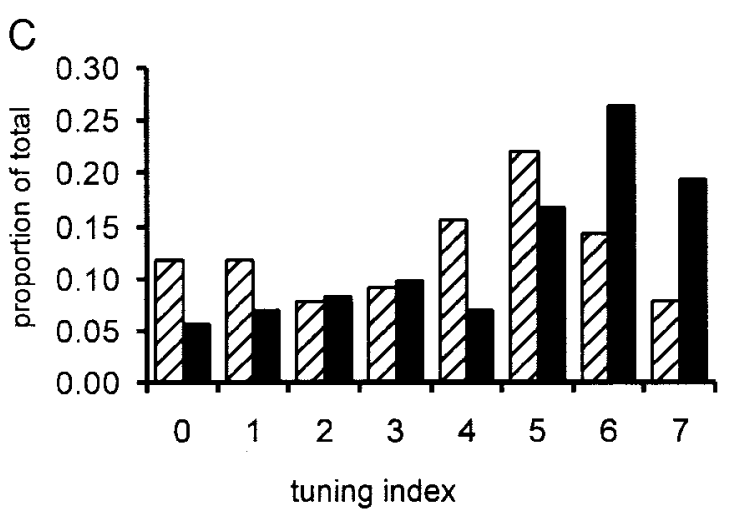

FIG. 3. Directional tuning of ON responses in VPM and PrV. All data shown here are for PW deflections only. A: normalized and rotated population polar plots for PrV and VPM reveal similar degree of ON response tuning within both populations. $B$ : distributions of tuning ratios, defined for each cell as average on response ( $n=8$ angles) divided by on response at maximally responsive angle. Small tuning ratios indicate pronounced directional tuning. Arrows point to bins containing mean values $($ solid $=\operatorname{PrV}$; dashed $=\mathrm{VPM})$. $C$ : distributions of tuning indices for PrV and VPM. Cells were assigned an index based on number of angles (0-7) that evoked responses statistically smaller than response at maximally responsive angle $(P<0.05$; one-tailed $t$-test). Index of 7 indicates highest degree of directional tuning.

comparing the plateau responses of VPM neurons to those of PrV. Cells in our study were classified as tonic if the best-angle plateau response significantly exceeded prestimulus firing rates $(P<0.025$; one-tailed $t$-test $)$. The remainder of neurons in a population were designated phasic by default. Figure $4 A$ reveals that the majority of both populations were classified as tonic, with a larger percentage of PrV cells (93\%; 67 of 72 cells) than VPM cells (74\%; 57 of 77 cells) falling into this category $\left(P<0.005 ; \chi^{2}\right.$ test $)$. In Fig. $4 B$ we compare the magnitudes and time courses of PrV and VPM population responses throughout the plateau duration of the stimulus. Overlaid population PSTHs reveal that, although VPM plateau activity was less robust than that of $\operatorname{PrV}$ at all time points, the difference in response magnitude was most pronounced during the initial $40 \mathrm{~ms}$ of the plateau response (only responses at the best angle were included in the PSTHs).

\section{AW-evoked excitation}

Previously we reported that, in lightly narcotized rats, VPMprojecting PrV neurons respond to deflections of multiple whiskers, with $\mathrm{AW}$-evoked responses occurring at longer latencies than PW-evoked responses (Minnery and Simons 2003). Here we investigated how the trigeminothalamic circuit transforms receptive-field structure by comparing the receptive fields of PrV cells with those of VPM neurons. We recorded responses to AW deflections for 60 of 77 VPM neurons $(n=$ 194 AWs) and all of $72 \mathrm{PrV}$ neurons $(n=197$ AWs). When possible, for each cell we stimulated the immediately adjacent rostral, caudal, ventral, and dorsal whiskers. In PrV, 71\% (139/197) of AWs yielded statistically significant on responses (see METHODS), whereas in VPM the percentage of responsive
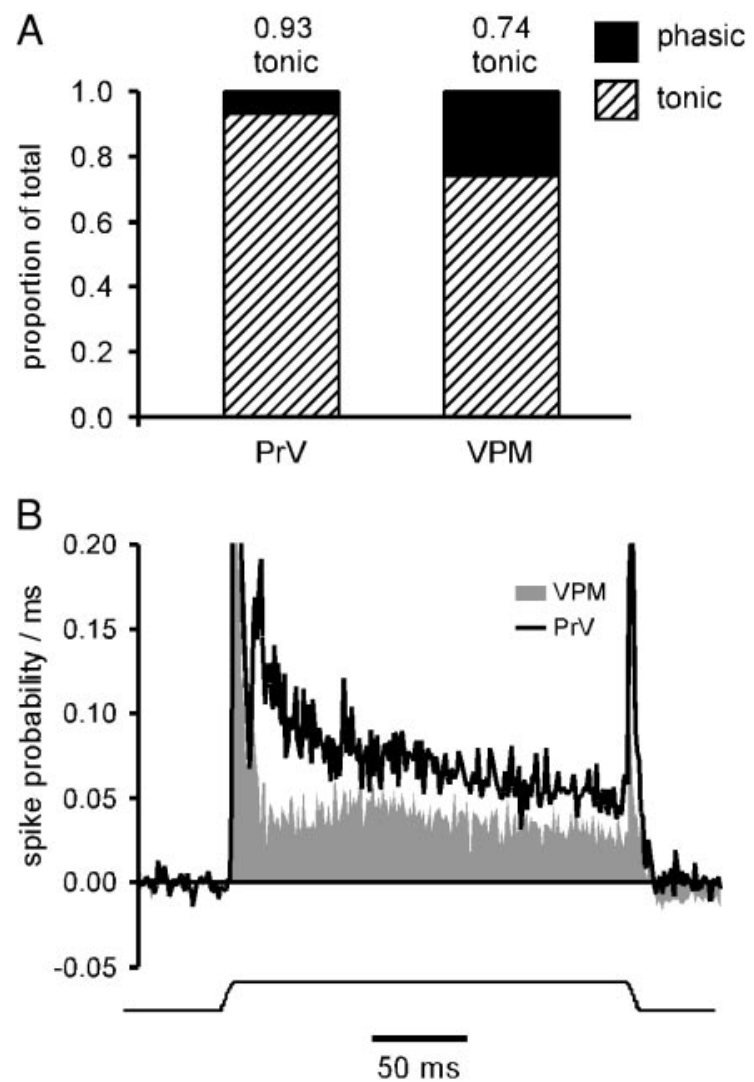

FIG. 4. Comparison of PrV and VPM plateau responses. A: proportion of cells in each population classified as tonic or phasic based on responses to PW deflections. Cells were classified as tonic if plateau activity at maximally responsive plateau angle significantly exceeded prestimulus activity levels $(P<0.025$; one-tailed $t$-test $)$. Cells that did not meet this criterion were classified as phasic. B: overlaid, background-subtracted population PSTHs showing plateau activity of tonic PrV and VPM neurons. Only data from each cell's maximally responsive plateau angle are included in PSTHs. ON and OFF responses are truncated. 
AWs, 39\% (75/194), was significantly lower $\left(P<0.0001 ; \chi^{2}\right.$ test). In both populations, the majority of cells expressed significant on responses for deflections of at least one $\mathrm{AW}$; however, the percentage of such multiwhisker neurons was greater in $\operatorname{PrV}$ (90\%; 65 of 72 cells) than in VPM $(73 \% ; 44$ of 60 cells; $P<0.05 ; \chi^{2}$ test). Mean on responses for significantly responsive AWs are listed in Table 2. Because we were not always able to test all four AWs for each cell, we did not attempt to calculate receptive-field size on a per-cell basis. For the same reason, the percentages of multiwhisker neurons reported here likely represent an underestimation of the true fraction of neurons in each population having multiwhisker receptive fields.

Figure 5 shows the population PSTHs for responsive AWs superimposed against the PSTHs of their corresponding PW populations. The top PSTHs, which illustrate data from all deflection angles, reveal that the spatial focus of receptive fields was strong within both nuclei despite the presence of significant AW responses. That is, the magnitudes of the PW population responses were large relative to those of AWs. However, comparison of AW and PW PSTHs at each whisker's maximally responsive angle (bottom PSTHs) indicates that the AW population response was relatively robust when stimulus parameters were optimal.

To quantify these observations, we divided each statistically significant AW ON response by that of its corresponding PW. The resulting AW:PW on ratio represents a measure of the relative magnitude of the $\mathrm{AW}$ response with respect to that of the PW (see also Fig. 7). For each AW-PW pair, this calculation was performed for both the best-angle on response $\left(\mathrm{ON}_{\max }\right)$ and the ON response averaged over all angles $\left(\mathrm{ON}_{\mathrm{all}}\right)$. Mean $\left( \pm\right.$ SD) AW:PW ratios for $\mathrm{ON}_{\max }$ were greater in VPM $(0.56 \pm 0.22)$ than in $\operatorname{PrV}(0.44 \pm 0.25)$, as was also the case for ratios calculated using $\mathrm{ON}_{\text {all }}(\mathrm{VPM}=0.44 \pm 0.20 ; \mathrm{PrV}=$ $0.39 \pm 0.24)$. In both cases, the differences between VPM and PrV were significant (both values of $P<0.05$; Mann-Whitney $U$ test). Thus, although statistically significant $\mathrm{AW}$ on re- sponses were more abundant in $\operatorname{PrV}$, the relative magnitudes of these responses were on average greater in VPM.

\section{AW-evoked inhibition}

Particular attention has been paid in recent literature to the origins of "cross-whisker" inhibition, or AW-evoked inhibition, within VPM (Brecht and Sakmann 2002; Desîlets-Roy et al. 2002; Pinault and Deschênes 1998a; Simons and Carvell 1989; Varga et al. 2002). Here we compared the extent to which PrV and VPM neurons manifest an exclusively inhibitory component to their surround receptive fields. We inferred the presence of $\mathrm{AW}$-evoked inhibition by identifying those AWs for which whisker deflection resulted in the suppression of activity below background levels. Although a given AW might conceivably elicit a combination of excitation and suppression, for the purposes of the present study we sought to examine specifically the phenomenon of suppression as it occurs independently of excitation.

First we selected from the AW data set those AWs that failed to evoke a significant on response at the best deflection angle. For these AWs, we then assessed whether activity levels at the minimally responsive on angle fell below prestimulus firing rates $(P<0.05$; one-tailed $t$-test $)$. We discarded from our analysis those cells having low $(<6 \mathrm{~Hz}$; see METHODS) spontaneous activity levels, given that sufficient spontaneous activity was necessary to detect suppression. Under these criteria, AW-evoked suppression was significantly more prevalent in VPM, where suppression was evoked by 84 of 194 AWs (43\%), than in PrV, where 19 of 197 AWs $(10 \%)$ elicited suppression $\left(P<0.005 ; \chi^{2}\right.$ test). Figure 6 shows the population PSTHs for those AW responses that met the criteria for suppression. For both cell populations, transient drops in activity levels in response to stimulus onset are readily apparent, even when all deflection angles are included in the PSTHs (Fig. 6, top).

To quantify suppression, the ratio of the ON response to spontaneous (prestimulus) activity was computed for each AW

TABLE 2. Adjacent whisker responses in $\operatorname{PrV}$ and VPM

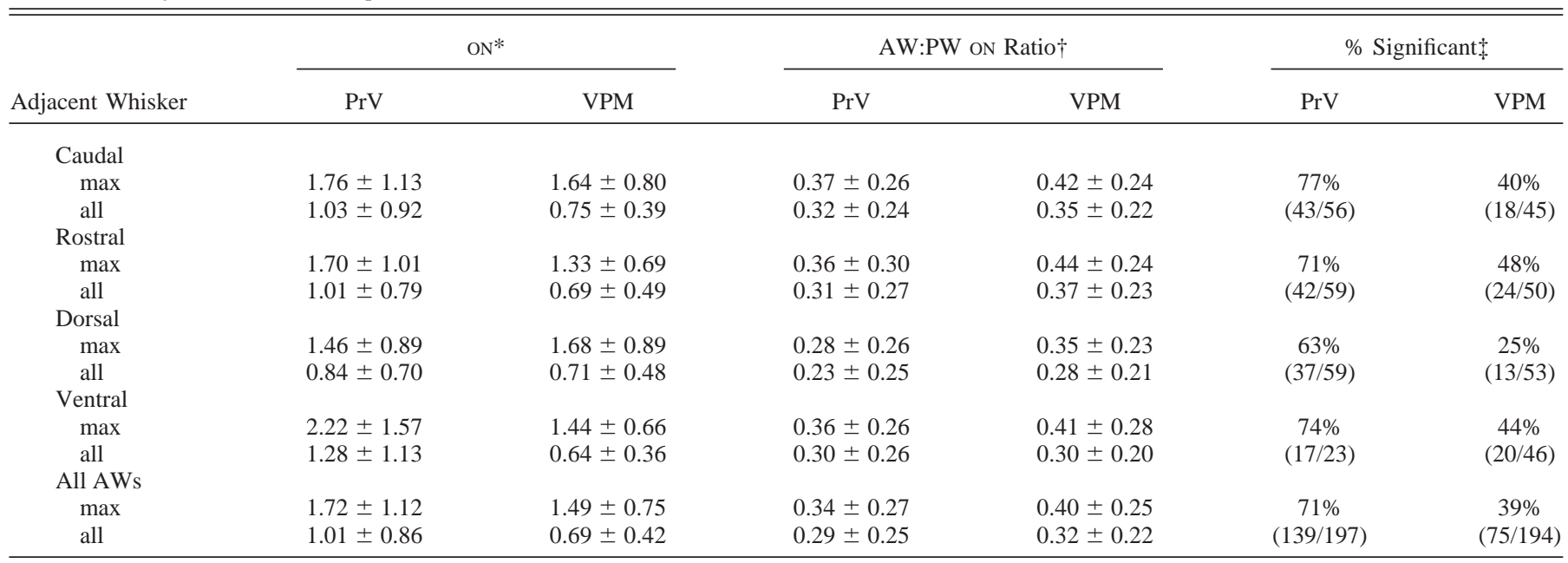

All values are mean \pm SD spikes per stimulus unless otherwise indicated. PW, principal whisker; AW, adjacent whisker; other abbreviations are as indicated in legend of Table 1. * Only statistically significant responses (see below) were used to compute means. $†$ For calculation of AW:PW ON ratios, each AW for a given cell was paired with the corresponding PW for that cell. Because all AWs were included in the calculations, mean ratios represent the net contribution of excitatory, inhibitory, and nonresponsive AWs (see RESULTS for AW:PW oN ratios calculated using only significant responses). $\ddagger$ Percentage of AWs eliciting statistically significant ON responses (see METHODS). Numbers in parentheses are significant AWs/total AWs. 

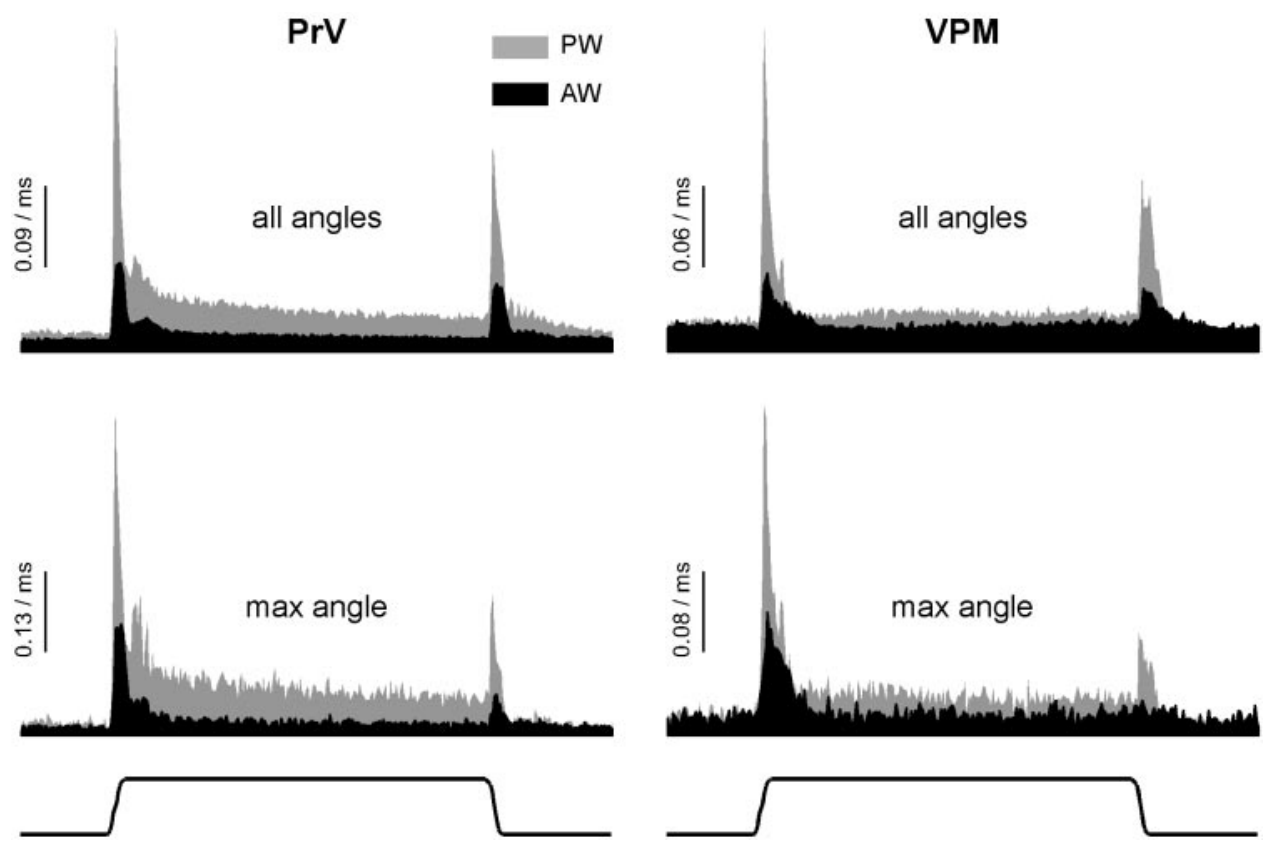

FIG. 5. Population PSTHs of adjacent (AW) and principal whisker (PW) responses in PrV and VPM. Only statistically significant AW responses $(P<0.05$; one-tailed $t$-test) and their corresponding PWs are included in PSTHs (PrV: $n=139$ AWs, 65 PWs; VPM: $n=75$ AWs, 44 PWs). "All angles": responses at all deflection angles are included; "max angle": maximally responsive on angle. Units on scale bars are spike probability/ms. PSTH bin width is 1 ms; total $=320$ bins for each PSTH. Rampand-hold stimulus waveforms are illustrated at bottom. classified as suppressive. This calculation was performed for both the minimally responsive oN angle ( $\mathrm{ON}_{\mathrm{min}} / \mathrm{Spont}$ ) and for the on response averaged over all angles ( $\left.\mathrm{ON}_{\text {all }} / \mathrm{Spont}\right)$. For both $\mathrm{PrV}$ and VPM, ON ${ }_{\min } /$ Spont ratios were small $\left(\mathrm{ON}_{\min } /\right.$ Spont $=$ $0.10 \pm 0.17$ for PrV; $0.17 \pm 0.18$ for VPM). This was anticipated given that spontaneous firing rates were often low to begin with, and selection for $\mathrm{ON}_{\min }$ in a cell with no excitatory response occasionally yielded zero spikes altogether. A more informative measure of suppression was provided by $\mathrm{ON}_{\mathrm{all}} /$ Spont, which for both nuclei was much larger than $\mathrm{ON}_{\mathrm{min}}$ / Spont, and in both cases exceeded $0.5\left(\mathrm{ON}_{\mathrm{all}} / \mathrm{Spont}=0.62 \pm\right.$ 0.28 for $\mathrm{PrV}$; $0.79 \pm 0.34$ for $\mathrm{VPM}$ ).

\section{Net contribution of $A W$ responses to receptive-field structure}

Given the presence of both excitatory and inhibitory AW responses in PrV and VPM receptive fields, we examined the net contribution of AW-evoked responses to a cell's receptive field. AW:PW on ratios were calculated for the entire population of AWs, including those AWs that failed to elicit statistically significant excitation and/or suppression. Figure $7 A$ reveals that, despite the contribution of AW-evoked suppression, AW responses were on average excitatory for both PrV and VPM. Remarkably, even though the relative prevalence of AW-evoked suppression versus excitation was greater in VPM than in PrV, average AW:PW ON ratios were slightly larger for the VPM population (values of $P<0.01$ for ratios calculated using both maximum ON and average on responses; Mann-Whitney $U$ test). This finding can likely be explained by two observations: 1) AW-evoked suppression in VPM, although widespread, was weak compared with excitation; 2) higher spontaneous firing rates of VPM neurons contributed to greater AW:PW ON ratios for the VPM population (see Table 1).

To assess whether the relative magnitudes of AW responses were related to the position (i.e., caudal, rostral, etc.) of the AW with respect to the PW, we computed mean

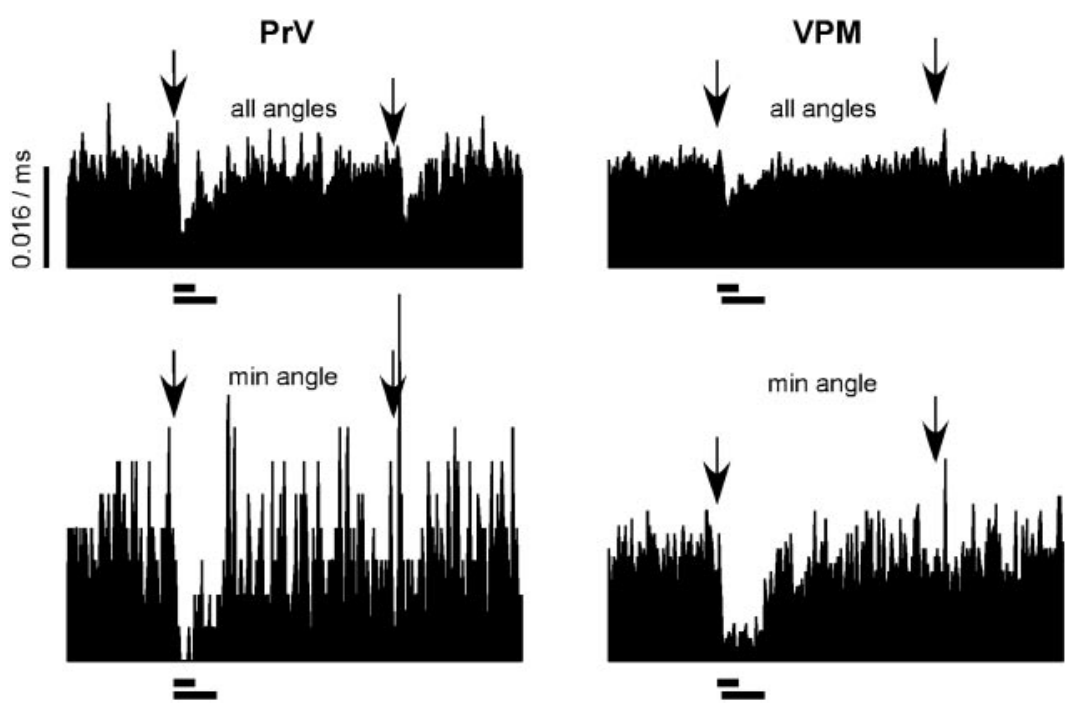

FIG. 6. AW-evoked activity suppression in PrV and VPM neurons. AW responses were classified as suppressive according to criteria described in METHODS. Bars under PSTHs indicate standard 20-ms oN window used to test for excitatory oN responses (short bar) and the longer, 40-ms on window (long bar) used to test for stimulus-evoked activity suppression. Arrows point to bins where excitatory ON and OFF responses would normally begin if present. "All angles": data from all deflection angles are included in PSTH. "Min angle": minimally responsive angle as determined by $40-\mathrm{ms}$ ON window. Units on scale bar are spike probability/ms. PSTH bin width is $1 \mathrm{~ms}$ (total $=421$ bins). PrV: $n=19$ AWs; VPM: $n=84$ AWs. 

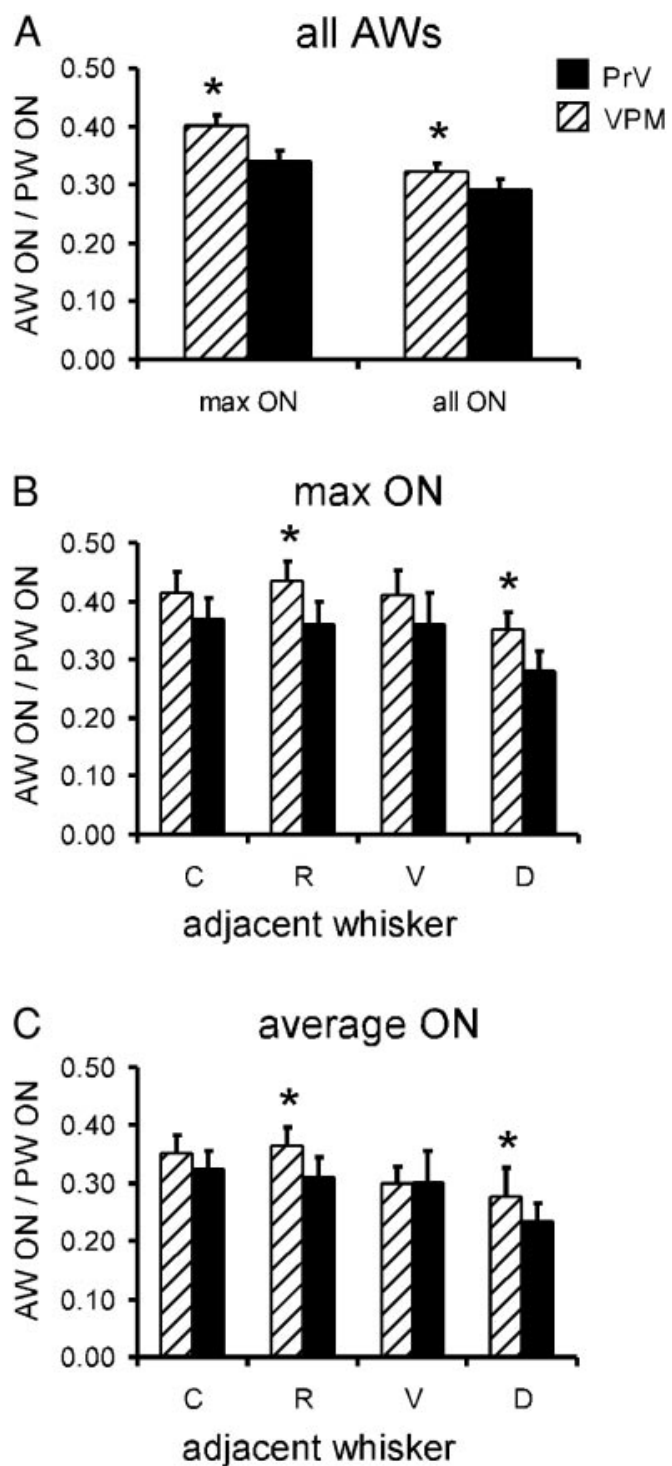

FIG. 7. Relative magnitude of AW responses. Each AW ON response was divided by ON response of its corresponding PW to yield an AW:PW ON ratio. A: mean AW:PW on ratios for all AWs (PrV: $n=197$; VPM: $n=194$ ). Data are presented separately for maximally responsive ON angle ("max ON") and ON responses averaged over all 8 angles ("average oN"). B: mean AW:PW ON ratios for each of $4 \mathrm{AWs}$ examined (C, caudal; R, rostral; $\mathrm{V}$, ventral; D, dorsal). Ratios in $B$ were calculated using maximally responsive angle for both PWs and AWs. $C$ : same as in $B$, but on responses were averaged over all 8 deflection angles. All error bars are +1 SEM. *Significant difference between PrV and VPM $(P<0.05$; Mann-Whitney $U$ test $)$. See Table 2 for exact numerical values and for numbers of whiskers examined per location (i.e., caudal, rostral, etc.).

ratios for each of the four AWs. Separate ratios were calculated for both the best-angle (Fig. 7B) and average (Fig. $7 C$ ) on responses (no consistent relationship was observed between directional tuning of AW and PW responses). Although the tendency in both PrV and VPM was for caudal and rostral AWs to evoke greater relative responses than dorsal and ventral whiskers, this trend did not reach significance (all values of $P>0.1$; ANOVA). Figure 7, $B$ and $C$, shows that, for each whisker, AW:PW on ratios were overall similar between PrV and VPM. For the rostral and dorsal whiskers, however, AW:PW on ratios were significantly larger in the VPM population than in the $\operatorname{PrV}$ population (values of $P<0.05$; Mann-Whitney $U$ test).

\section{Temporal properties of $A W v s . P W$ responses}

Recent experimental and modeling studies indicate that cortical barrel circuitry is preferentially sensitive to initial firing synchrony among thalamic neurons (reviewed in Miller et al. 2001; see also DISCUSSION), with highly synchronous inputs producing large magnitude (total spike count) responses among cortical neurons. In a population PSTH, initial firing synchrony is represented by the degree of temporal dispersion in the early part of the PSTH. More synchronous responses exhibit less temporal dispersion and faster-rising initial slopes. Because response magnitude differences between $\mathrm{PW}$ and $\mathrm{AW}$ responses are greater in cortical barrels than in thalamic barreloids (i.e., cortical neuron responses are more focused on the PW than are thalamic responses), we investigated whether the temporal profiles of PW responses differ from those of AWs within the subcortical pathway.

Figure $8 A$ shows normalized, high-resolution $(100-\mu$ s bin width) ON and OFF PSTHs for each nucleus. Two features of the PSTHs are particularly noteworthy. First, AW and PW population responses had overall similar onset times except for the case of VPM OFF responses, wherein AW responses were delayed relative to those of PWs. Second, for both nuclei and both response windows, PW population responses were found to exhibit greater initial slopes and less temporal dispersion than those of AWs. To quantify the temporal dispersion of the population response, we computed for each PSTH the time to the 50th percentile spike $\left(\mathrm{T}_{50 \%}\right)$ within the response window. For each nucleus and each response window, the $\mathrm{T}_{50 \%}$ for the AW population response was greater than that of the PW response (see Fig. 8A for population $\mathrm{T}_{50 \%}$ values). We also calculated $\mathrm{T}_{50 \%}$ values for the responses of single neurons (Table 3). Again, for each nucleus and each response window, the mean $\mathrm{T}_{50 \%}$ for $\mathrm{AW}$ responses was found to exceed that of PWs (all values of $P<0.05$; Mann-Whitney $U$ test).

If initial firing synchrony is greater in the PW than in the AW populations, then one would expect less variation, or less temporal dispersion, in the latency to onset of PW responses versus AW responses. We analyzed response latency by two methods. First, for each deflected whisker we determined the mean latency to the first spike (100- $\mu$ s temporal resolution) within the response window (see METHODS). We then averaged these mean latencies across the population. We found that the variation in mean latency to first spike, as expressed by the SD for the population, was indeed greater among the AW than the PW population (Table 3). Also, mean latencies were on average greater for $\mathrm{AW}$ than for $\mathrm{PW}$ responses, indicating a greater contribution of longer latency responses to the AW population response. The difference between mean AW and PW latencies was significant in both nuclei for both on and off windows (all values of $P<0.05$; Mann-Whitney $U$ test).

As a second measure of latency, we identified response onset for each individual PSTH as the first of two consecutive 1-ms bins for which activity significantly exceeded prestimulus levels (see METHODS; see also Minnery and Simons 2003). For this method, latency was defined relative to the onset of the stimulus as opposed to the start of the response window and so represents absolute latency to response onset. Again we found 
A
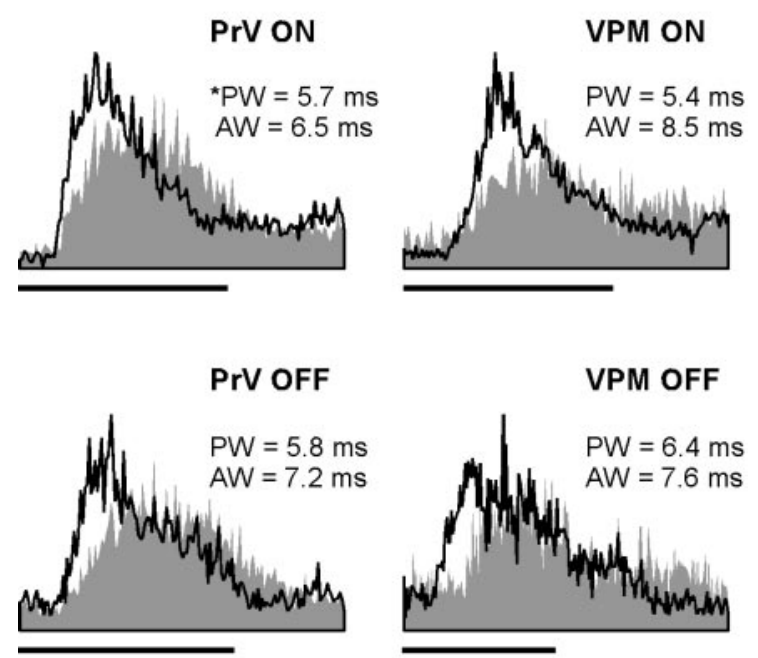

B

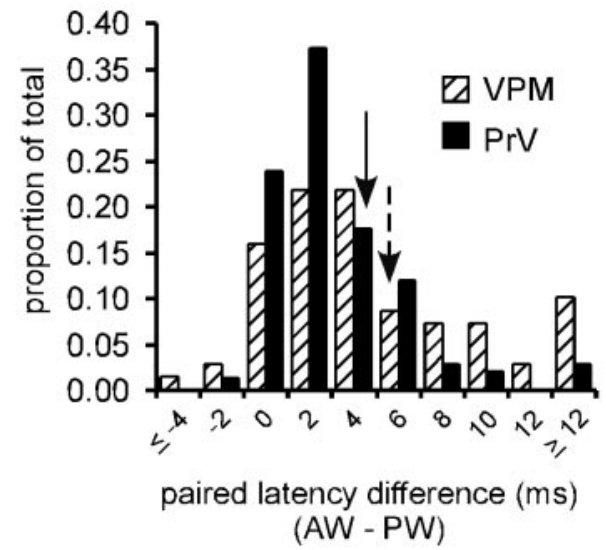

FIG. 8. Temporal structure and latency differences of AW and PW responses in PrV and VPM. A: normalized, high-resolution (100- $\mu$ s bin width) PSTHs of ON and OFF responses reveal that for both PrV and VPM populations, AW population responses have slower rise times and are more temporally dispersed than those of PWs. *Numbers above PSTHs are population $\mathrm{T}_{50 \%}$ values. Only statistically significant responses $(P<0.05$; one-tailed $t$-test $)$ are included in each PSTH (see Table 3 legend for numbers of whiskers per population PSTH). Scale bars $=10 \mathrm{~ms}$. B: distribution of AW-PW paired latency differences in PrV and VPM. To compute paired latency differences, each AW was matched with its corresponding PW, and difference in response latencies (AW $-\mathrm{PW}$ ) was calculated (PrV: $n=142$; VPM: $n=69$ ). Arrows indicate bins containing mean values $($ solid $=\operatorname{PrV}$; dashed $=$ VPM $)$.

that response latency among the AW population was more variable than that for PWs (see SDs in Table 3). Also, as was the case with latency to first spike, the mean response latency for PWs was less than that for AWs (Table 3), with the difference in mean latencies being greater in VPM than in PrV (difference $=4.9 \mathrm{~ms}$ for VPM, $2.4 \mathrm{~ms}$ for PrV). For both nuclei, differences between the mean response latencies of AWs and PWs were statistically significant (values of $P<$ 0.0001; Mann-Whitney $U$ test).

Previous studies of VPM neurons have shown that, for cells with multiwhisker receptive fields, AW responses occur on average at longer latencies than PW responses (ArmstrongJames and Callahan 1991; Diamond et al. 1992; Friedberg et al. 1999; Lee et al. 1994a). To assess the extent to which the AWPW latency differential was present for individual neurons in our study, we computed paired latency differences, defined as the difference in latency between each AW response and that of its corresponding PW. The distributions of paired latency differences for PrV and VPM are shown in Fig. 8B. The data clearly show that, for cells in both nuclei, AW responses occurred at longer latencies than those of their matched PWs. Mean ( \pm SD) paired latency differences for PrV and VPM were, respectively, $2.5 \pm 3.6$ and $4.9 \pm 6.0 \mathrm{~ms}$. Although these means differed significantly between PrV and VPM $(P<$ 0.005; Mann-Whitney $U$ test), the modes of the histograms were similar. The VPM distribution, however, contained more long-latency outliers than the PrV distribution.

\section{I S C U S S I O N}

In the present study, we sought to characterize the nature of the trigeminothalamic response transformation within the lemniscal pathway by recording and comparing stimulus-evoked responses of single neurons within both pre- (PrV) and postsynaptic (VPM) populations. Our data indicate that VPM responses, although similar in several respects to those of $\mathrm{PrV}$, differ markedly from their presynaptic counterparts along a number of parameters. This suggests that synaptic mechanisms and/or network interactions function to transform the subcortical afferent signal at the level of the diencepahlon.

\section{Signal transmission at the $\operatorname{PrV} \rightarrow$ VPM synapse}

Comparison of PrV and VPM responses at both the singleunit and population levels reveals substantial similarities between pre- (PrV) and postsynaptic (VPM) populations. In particular, both PrV and VPM neurons respond vigorously to the transient ON and OFF phases of the stimulus, and less robustly during the stimulus plateau. Our finding that VPM cells express many of the response characteristics of their PrV inputs is consistent with recent in vitro data indicating that the afferent input to a given VPM cell is dominated by a single lemniscal fiber (Castro-Alamancos 2002a). For example, the strong similarities in directional tuning between PrV and VPM cells may be attributable to the direct transmission of tuning properties from individual PrV neurons to their postsynaptic targets. Although future anatomical studies will be required to resolve in detail the convergence/divergence patterns of PrV axons onto VPM cells, it appears that the organization of the lemniscal trigeminothalamic pathway in the rat may parallel that of the retinogeniculate system in other species, where relay neurons of the lateral geniculate nucleus (LGN) receive synapses from only a small number of retinal ganglion cells (Mastronarde 1987, 1992; Usrey et al. 1999). Interestingly, LGN neurons, like those of VPM, also exhibit response properties and receptive-field structures similar to their inputs (but see Humphrey and Weller 1988 for evidence of temporal processing in the LGN).

One striking difference between the PrV and VPM populations, however, is the decrease in response magnitude relative to spontaneous activity levels (signal:background) in VPM with respect to PrV. This reduction in relative signal strength is attributed to both the smaller response magnitudes and greater spontaneous firing levels of VPM neurons. Although Rt-mediated thalamic inhibition might account for some of the reduction in VPM response magnitudes, it is unlikely to fully explain 
TABLE 3. Temporal distribution and response latencies of adjacent and principal whisker responses in PrV and VPM

\begin{tabular}{|c|c|c|c|c|c|c|}
\hline Neuron & PW & AW & PW & AW & PW & AW \\
\hline $\mathrm{ON}$ & $5.79 \pm 2.75$ & $6.74 \pm 3.28^{*}$ & $3.76 \pm 1.82$ & $6.41 \pm 2.46^{*}$ & $4.04 \pm 1.66$ & $6.46 \pm 3.95 *$ \\
\hline OFF & $5.72 \pm 2.73$ & $7.54 \pm 2.83^{*}$ & $4.69 \pm 2.00$ & $7.17 \pm 2.23^{*}$ & - & - \\
\hline \multicolumn{7}{|l|}{ VPM } \\
\hline ON & $5.61 \pm 2.96$ & $8.70 \pm 3.17 *$ & $4.28 \pm 1.79$ & $7.90 \pm 2.55^{*}$ & $5.40 \pm 1.70$ & $10.30 \pm 6.30 *$ \\
\hline
\end{tabular}

All values are mean \pm SD ms. $\S \mathrm{T}_{50 \%}$ is the time to the 50th percentile spike within the given response window. $\dagger$ Latency to occurrence of the first spike within the response window was measured at $100-\mu \mathrm{s}$ temporal resolution for each trial for which at least one spike was present, and all latencies were then averaged to compute a mean latency per whisker. These mean latencies were then used to compute population means for both PW and AW responses. $\$$ Latency to response onset was computed with respect to the onset of the stimulus, and was defined as the first of two consecutive 1-ms bins in which the number of spikes significantly exceeded prestimulus activity $(P<0.05)$. * Difference between AWs and PWs is statistically significant $(P<0.05$ Mann-Whitney $U$ test $)$. All values presented here are based on responses at all 8 deflection angles. For $\mathrm{T}_{50 \%}$ and latency-to-first-spike measurements, only statistically significant AW responses $(P<0.05$; one-tailed $t$-test $)$ and their corresponding significant PW responses are included in the calculations (PrV ON: $n=65$ PWs, 139 AWs; PrV OFF: $n=61$ PWs, 141 AWs; VPM ON: $n=44$ PWs, 75 AWs; VPM OFF: $n=38$ PWs, 68 AWs). For latencies to response onset, only AWs with identifiable response latencies and their corresponding PWs are included in calculations of average latencies (PrV: $n=67$ PWs, 142 AWs; VPM: $n=45$ PWs, $69 \mathrm{AWs})$.

the smaller magnitudes of VPM ON responses, given that oN responses begin before the engagement of strong feedback inhibition. It is conceivable that high spontaneous firing rates among the Rt population (Hartings et al. 2000) provide a tonic barrage of hyperpolarizing inputs to VPM neurons, thereby rendering them less responsive to input from PrV; however, two observations argue against this interpretation. First, the high spontaneous activity of VPM cells in our study suggests that these neurons were not strongly hyperpolarized. Second, Hartings and Simons (2000) showed under similar experimental conditions that blockade of GABAergic inhibition onto individual VPM neurons fails to increase peak firing rates of oN responses.

It is probable therefore that the observed decrease in response magnitude along the PrV $\rightarrow$ VPM pathway is attributable in part to synaptic constraints and/or circuit mechanisms other than feedback inhibition. For example, strong synaptic depression of excitatory postsynaptic potentials (EPSPs) has been shown to occur at the PrV $\rightarrow$ VPM synapse in vivo (Castro-Alamancos 2002b) as well as in vitro (Castro-Alaman$\cos 2002 \mathrm{a})$. The effects of such depression might be pronounced under conditions of high spontaneous activity, given that a stimulus-evoked spike is more likely to occur in close temporal proximity to a preceding spontaneous spike. Regardless of its origins, however, the decrease in absolute response magnitude from PrV to VPM appears to represent a trend in the whisker-to-barrel pathway because similar reductions in stimulus-evoked spike counts have been shown to occur within the $\mathrm{NV} \rightarrow$ PrV (Minnery and Simons 2003) and VPM $\rightarrow$ SI (Bruno and Simons 2002; Castro-Alamancos and Oldford 2002; Simons and Carvell 1989) circuits.

\section{Implications for coding strategies}

A decrement in signal:background ratios along the subcortical afferent pathway might at first seem disadvantageous for sensory processing. However, recent models of barrel cortex circuitry suggest that the temporal structure of the VPM population response, rather than its magnitude, is most critical in determining the strength of the cortical response (Kyriazi and Simons 1993; Pinto et al. 1996, 2000; Simons 1997). Specif- ically, both physiological and modeling data demonstrate a preference by the layer IV cortical circuit for strong initial firing synchrony among its inputs (VPM), which in the VPM population PSTH is evidenced by a fast rate of increase, or minimal temporal dispersion, in the early component of the response.

In the present study we find that, despite differences in response magnitudes between PrV and VPM populations, the temporal properties of responses are overall similar between the two nuclei (compare PrV and VPM population $\mathrm{T}_{50 \%}$ values in Fig. 8). Of particular interest is our finding that, within each nucleus, the early part of the population response is more temporally dispersed for the AW than for the PW population. Our data therefore indicate that the aspect of the subcortical afferent signal that is most relevant to the cortical circuit, i.e., its temporal structure, is preserved across the PrV $\rightarrow$ VPM synapse. This result further suggests that the small receptive fields observed in barrel cortex neurons (Simons and Carvell 1989) may result at least in part from differences in the temporal structure of PW versus AW inputs, and that these differences emerge during the earliest stages of multiwhisker integration within the subcortical pathway.

The mechanisms underlying temporal differences in response profiles within populations-e.g., ON-versus-OFF and $\mathrm{AW}$-versus-PW-are unclear. In the case of $\mathrm{ON}$ and $\mathrm{OFF}$ responses, a slight difference in temporal dispersion is apparent within PrV but becomes abruptly more pronounced at the level of VPM, where ofF responses are less temporally focused than on responses (see also Fig. 8 in Brecht and Sakmann 2002 for an example of greater temporal dispersion in VPM OFF responses). As suggested previously by Kyriazi and colleagues (1994), the OFF-ON temporal profile difference may be related to a differential contribution of $I_{T}$-mediated rebound spikes to the ofF response, although at present this issue requires further examination. As for the temporal differences between $\mathrm{AW}$ and $\mathrm{PW}$ population responses, Brecht and Sakmann (2002) recently showed using intracellular recordings in vivo that, for a prominent subclass of multiwhisker VPM neurons, AW-evoked EPSPs occur at longer and more variable latencies than those evoked by PW deflections. This difference was in turn 
shown to be reflected as a greater temporal dispersion in spike output.

\section{Response adaptation}

Previous studies have suggested that the expression of plateau responses within the VPM population is regulated by intrinsic thalamic circuitry, with inhibitory feedback from Rt functioning to suppress the effects of sustained, or tonic, inputs arriving from PrV (Gottschaldt et al. 1983; Hartings and Simons 2000; Lee et al. 1994a,b). However, the extent of this regulation has been difficult to determine because the adaptive properties of VPM-projecting PrV neurons themselves have only recently been studied in detail (Chiaia et al. 2000; Minnery and Simons 2003) and may depend critically on the types of stimuli and anesthesia employed in a given study (see DISCUSSION in Minnery and Simons 2003). We show here that, under similar experimental and stimulus conditions, fewer VPM than PrV neurons manifest statistically significant plateau responses. Furthermore, the time course of plateau activity differs markedly during the early phase of the response. Whereas PrV plateau responses are strongest during the early phase, VPM plateau activity starts out at low levels after the termination of the on response but then increases gradually. The duration of this early reduction in VPM plateau activity is similar to the width of the Rt population ON response (about 35 ms) recorded under identical conditions (Hartings et al. 2000) and also similar in length to the time window over which AWand/or PW-evoked inhibition in VPM is reported to be maximal (Simons and Carvell 1989). When taken in context with these earlier studies, the magnitude and time course of the PrV-VPM plateau response differential seen here are consistent with an Rt-mediated inhibitory influence on VPM firing patterns.

\section{Receptive-field synthesis in the trigeminothalamic pathway}

The present findings shed new light on an unresolved question regarding the origin of multiwhisker receptive fields in VPM. Although early studies of VPM neurons described primarily single-whisker receptive fields (Chiaia et al. 1991b; Rhoades et al. 1987; Shosaku 1985; Sumitomo and Iwama 1987; Vahle-Hinz and Gottschaldt 1983; Waite 1973), a consensus has emerged in recent years that VPM cells recorded under light anesthesia respond to deflections of multiple whiskers (Armstrong-James and Callahan 1991; Diamond et al. 1992; Friedberg et al. 1999; Simons and Carvell 1989), and that AW responses occur at longer latencies than those of PWs (see preceding references, and see also Brecht and Sakmann 2002). However, the origin of these multiwhisker responses remains a source of controversy. One possibility is that dendrites of VPM neurons, which are known to extend beyond the dimensions of a single barreloid (Chiaia et al. 1991b; Harris 1986; Ohara and Havton 1994; Varga et al. 2002), integrate information from lemniscal inputs originating in nonhomologous barrelettes. Yet recent ultrastructural analyses of VPM dendrites by Varga et al. (2002) indicate that lemniscal synapses onto these extra-barreloid dendrites are rare if not absent altogether.

Another possibility is that corticothalamic feedback excitation contributes to AW responses in VPM. Forty-six percent of
VPM AW responses in our study occurred at latencies more than $3 \mathrm{~ms}$ longer than those of their corresponding PW responses. Assuming an average thalamocortical-corticothalamic conduction time of approximately $4 \mathrm{~ms}$ (Bruno and Simons 2002; Kelly et al. 2001), it is theoretically possible that some of the long-latency AW responses in VPM arise from corticothalamic feedback. However, prevailing physiological evidence argues against a corticothalamic origin for AW responses in VPM. In particular, several studies have shown that most corticothalamic neurons in layer VI, where the SI $\rightarrow$ VPM projection originates, are unresponsive to stimuli when examined under passive conditions (Landry and Dykes 1985; Swadlow 1989; Swadlow and Hicks 1996), and that even when such neurons are activated pharmacologically, a somatotopically precise barrel-to-barreloid connectivity pattern is revealed (Temereanca and Simons 2001). Less is known about corticofugal inputs to PrV. However, given the comparatively small AW-PW latency differentials observed in $\operatorname{PrV~}(71 \%, \leq 3$ $\mathrm{ms}$ ), it is unlikely that a substantial fraction of $\operatorname{PrV}$ AW responses result from cortical feedback, especially because such a signal must traverse at least three additional synapses before returning to $\operatorname{PrV}$.

Several groups have argued that the longer-latency AW responses observed in VPM reflect input from the spinal trigeminal nucleus $(\mathrm{SpV})$, specifically from subnucleus interpolaris (SpVi) (Armstrong-James and Callahan 1991; Friedberg et al. 1999; Lee et al. 1994a; Rhoades et al. 1987). On the one hand, these arguments seem plausible given the well-established existence of a trigeminothalamic pathway originating in SpVi (Chiaia et al. 1991a; Erzurumlu and Killackey 1980; Peschanski 1984; Pierret et al. 2000; Veinante et al. 2000; Williams et al. 1994), and given that thalamic-projecting SpVi neurons themselves possess multiwhisker receptive fields (Jacquin et al. 1986, 1989). Furthermore, SpVi axons innervating VPM have slower conduction velocities than those of fibers originating in PrV (Veinante et al. 2000). This latter finding has been proposed to account for longer $\mathrm{AW}$ response latencies in VPM. On the other hand, recent anatomical evidence suggests that $\mathrm{SpVi}$ and $\mathrm{PrV}$ project to nonoverlapping regions within VPM. Whereas PrV axons innervate the dorsomedial portion of VPM and target the barreloid "core," SpVi terminals are sparse and are relegated to the ventral lateral portion of VPM, where barreloids taper into cytochrome oxidase-poor "tails" (Pierret et al. 2000; Williams et al. 1994). In light of these latter studies, it appears unlikely that $\mathrm{SpVi}$ inputs alone can account for the widespread presence of multiwhisker receptive fields in VPM.

Altogether, our comparisons of receptive-field properties between PrV and VPM neurons suggest that PrV inputs are sufficient to account for most of the major organizational features of VPM receptive fields. Previously we reported that, in lightly narcotized rats, the majority of PrV neurons respond to deflections of at least one AW (Minnery and Simons 2003). Here we show that the relative magnitude of AW with respect to PW responses is similar in VPM and PrV. Moreover, we find that AW responses within both populations occur at longer latencies than PW responses, and that AW population responses are more temporally dispersed than those of PWs. Our findings therefore add complementary physiological data to the growing body of anatomical evidence suggesting that $\operatorname{PrV}$ 
constitutes the overwhelming source of afferent drive to barreloid neurons.

Based on our data, we cannot exclude the possibility that inputs from $\mathrm{SpVi}$ do in fact contribute to stimulus-evoked activity in VPM, particularly because late-arriving signals from $\mathrm{SpVi}$ might have been missed by our latency analyses. Indeed, the greater proportion of large AW-PW latency differentials observed in VPM might be attributed to SpVi inputs. However, it is most probable that any interactions between $\mathrm{SpV}$ and the lemniscal pathway occur first within the brain stem itself, where a dense plexus of intersubnuclear connections links PrV with each of the three SpV subnuclei (Haring et al. 1990; Jacquin et al. 1990). These intersubnuclear fibers may themselves provide the substrate for multiwhisker receptive-field synthesis within PrV (see DISCUSSION in Minnery and Simons 2003 for additional explanations of receptive-field structure in PrV neurons).

One surprising finding reported here is that fewer AWs evoke significant responses in VPM than in PrV. Weaker surround responses in VPM might simply result from the nonlinear thresholding of weak AW inputs compared with strong PW inputs. Another possibility is that more AW responses are present in VPM than are indicated by our analyses, but that high prestimulus firing rates among the VPM population prevent some responses from reaching statistical threshold. Also, it is conceivable that the occasional accidental recording of posterior group (PO)-projecting PrV cells may have led us to overestimate the prevalence of AW responses in PrV. PO-projecting PrV neurons, which are thought to possess large, multiwhisker receptive fields (Veinante and Deschênes 1999), might have been antidromically activated if stimulus currents occasionally spread beyond VPM into neighboring PO. However, we believe that contamination of our PrV sample by a few PO-projecting neurons cannot account for our observation that AW responses are more prevalent in PrV than in VPM, given that differences between the two nuclei were large and highly statistically significant $(P<0.0001$; see RESULTS). Although the misidentification of a larger number of PO-projecting neurons could theoretically have impacted our results, this is unlikely to have occurred. As discussed in detail in Minnery and Simons (2003) (see METHODs section in that study), antidromic stimulus currents were likely well-confined within VPM, given that close somatotopic alignment between stimulation and recording electrodes was typically required before antidromic responses could be evoked in PrV (see METhods in Minnery and Simons 2003 for additional details).

\section{A role for inhibition in subcortical sensory processing}

Here we provide evidence of AW-evoked suppression of background activity in both PrV and VPM cells. Four main conclusions can be drawn from our data: 1) AW deflections can suppress ongoing activity in both pre- (PrV) and postsynaptic (VPM) populations; 2) response suppression can occur independent of excitation; 3) AW-evoked suppression is more prevalent within VPM than in PrV; 4) classically defined lateral inhibition, as evidenced by AWevoked suppression without excitation (see, e.g., Kuffler 1953), is a relatively weak phenomenon in the lemniscal trigeminothalamic pathway and does not, on average, provide a counterbalance to $\mathrm{AW}$-evoked excitation. This last conclusion is derived from our data (Fig. 7) showing that AW-evoked responses are on average excitatory. This is even the case in VPM, where more AW-evoked responses are classified as suppressive than excitatory.

Although our data argue against a prominent role for lateral inhibition in the subcortical processing of spatial information, our findings are nevertheless consistent with recent anatomical accounts of thalamic inhibitory circuitry. Desîlets-Roy et al. (2002) showed that a thalamic barreloid representing a given whisker receives inhibitory inputs from Rt neurons whose receptive fields correspond to that same whisker, thus forming a topographically closed feedback loop. Within a barreloid, Rt axons synapse onto the cell bodies and proximal dendrites of neurons within the parent barreloid, as well as onto the distal dendrites of neurons whose cell bodies are located in adjacent barreloids (Varga et al. 2002). This arrangement provides one possible explanation as to how AW-evoked suppression could occur without excitation, because those same distal dendrites that receive $\mathrm{AW}$-evoked inhibition appear not to receive excitatory synapses from lemniscal (PrV) axons (Varga et al. 2002). Furthermore, the relatively weak suppression observed in VPM might be explained by the strong electrotonic filtering of inhibitory inputs onto distal dendrites.

The findings of the present study also agree with previous studies from our laboratory showing that stimulation of multiple AWs results in a net excitatory response in VPM neurons (Brumberg et al. 1996; Kelly et al. 1999; Simons and Carvell 1989). In one of these studies (Simons and Carvell 1989), a "test" stimulus consisting of a PW deflection was preceded at a brief interval by a "conditioning" stimulus applied to either an AW or to the PW itself. Both conditioning stimuli tended to suppress the response to the test stimulus, but the conditioning stimulus applied to the PW resulted in greater suppression on average than did the conditioning stimulus applied to the AW. These findings complement data from the present study, which demonstrate a weak inhibitory surround in VPM receptive fields and at the same time provide support for the notion of topographically "closed" inhibitory feedback loops in the intrathalamic circuit (see above).

Inferential evidence from our present data suggests that the effects of feedback inhibition on signals transiting VPM are most pronounced during the static (plateau) phase of the stimulus (Fig. 4). This result is not surprising, given that Rt neurons themselves exhibit strong tonic responses to sustained whisker displacements (Hartings et al. 2000). Additional studies of Rt neurons show that, despite their robust plateau responses, most Rt cells follow high-frequency periodic stimuli with comparatively less temporal fidelity (Hartings 2000). In other words, Rt responses represent a low-pass filtered version of the afferent signal. By extension, Rt neurons may confer high-pass properties on their VPM targets by subtracting out the slower components of the VPM response (Hartings 2000). In vitro and in vivo studies indicate that Rt feedback does indeed regulate responses of VPM neurons to high-frequency stimulus trains, with the fidelity of transmission varying according to arousal levels (Castro-Alamancos 2002a,b). Moreover, recent investigations into the influence of behavioral state on thalamic processing suggest that bottom-up neuromodulatory inputs from the brain stem may act in concert with top-down corticotha- 
lamic projections to influence the balance of excitation and inhibition in VPM, thereby actively regulating the responses of VPM neurons to their PrV inputs (reviewed in Nicolelis and Fanselow 2002).

We are grateful to H. Kyriazi and A. Myers for expert technical assistance.

\section{IS C L OS URES}

This work was supported by National Institute of Mental Health Grant MH-61372.

\section{REFERENCES}

Ahissar E, Sosnik R, and Haidarliu S. Transformation from temporal to rate coding in a somatosensory thalamocortical pathway. Nature 406: 302-306, 2000 .

Armstrong-James M and Callahan CA. Thalamo-cortical processing of vibrissal information in the rat. II. Spatiotemporal convergence in the thalamic ventroposterior medial nucleus (VPm) and its relevance to generation of receptive fields of S1 cortical "barrel" neurones. J Comp Neurol 303: 211-224, 1991.

Barbaresi P, Spreafico R, Frassoni C, and Rustioni A. GABAergic neurons are present in the dorsal column nuclei but not in the ventroposterior complex of rats. Brain Res 382: 305-326, 1986.

Bennett-Clarke CA, Chiaia NL, and Rhoades RW. Differential expression of acetylcholinesterase in the brainstem, ventrobasal thalamus, and primary somatosensory cortex of perinatal rats, mice, and hamsters. Somatosens Mot Res 16: 269-279, 1999.

Brecht M and Sakmann B. Whisker maps of neuronal subclasses of the rat ventral posterior medial thalamus, identified by whole-cell voltage recording and morphological reconstruction. $J$ Physiol 538: 495-515, 2002.

Brumberg JC, Pinto D, and Simons DJ. Spatial gradients and inhibitory summation in the rat whisker barrel system. J Neurophysiol 76: 130-140, 1996.

Bruno RM and Simons DJ. Feedforward mechanisms of excitatory and inhibitory cortical receptive fields. J Neurosci 22: 10966-10975, 2002.

Castro-Alamancos MA. Properties of primary sensory (lemniscal) synapses in the ventrobasal thalamus and the relay of high-frequency sensory inputs. J Neurophysiol 87: 946-953, 2002a.

Castro-Alamancos MA. Different temporal processing of sensory inputs in the rat thalamus during quiescent and information processing states in vivo. J Physiol 539: 567-578, 2002b.

Castro-Alamancos MA and Oldford E. Cortical sensory suppression during arousal is due to the activity-dependent depression of thalamocortical synapses. J Physiol 541: 319-331, 2002.

Chiaia NL, Rhoades RW, Bennett-Clarke CA, Fish SE, and Killacky HP. Thalamic processing of vibrissal information in the rat. I. Afferent input to the medial ventral posterior and posterior nuclei. J Comp Neurol 314: 201216, 1991b.

Chiaia NL, Rhoades RW, Fish SE, and Killackey HP. Thalamic processing of vibrissal information in the rat. II. Morphological and functional properties of medial ventral posterior nucleus and posterior nucleus neurons. J Comp Neurol 314: 217-236, 1991a.

Chiaia NL, Zhang S, Crissman RS, and Rhoades RW. Effects of neonatal axoplasmic transport attenuation on the response properties of vibrissaesensitive neurons in the trigeminal principal sensory nucleus of the rat Somatosens Mot Res 17: 273-283, 2000.

Crabtree JW, Collingridge GL, and Isaac TR. A new intrathalamic pathway linking modality-related nuclei in the dorsal thalamus. Nat Neurosci 1: 389_ 394, 1998.

Deschênes M, Veinante $\mathbf{P}$, and Zhang $\mathbf{Z W}$. The organization of corticothalamic projections: reciprocity versus parity. Brain Res Rev 28: 286-308, 1998.

Desîlets-Roy B, Varga C, Lavallee P, and Deschênes M. Substrate for cross-talk inhibition between thalamic barreloids. J Neurosci 22: 1-4, 2002.

Diamond ME, Armstrong-James M, Budway MJ, and Ebner FF. Somatic sensory responses in the rostral sector of the posterior group (POm) and in the ventral posterior medial nucleus (VPM) of the rat thalamus: dependence on the barrel field cortex. J Comp Neurol 319: 66-84, 1992.

Erzurumlu RS and Killackey HP. Diencephalic projections of the subnucleus interpolaris of the brainstem trigeminal complex in the rat. Neuroscience 5: 1891-1901, 1980.
Fanselow EE and Nicolelis MAL. Behavioral modulation of tactile responses in the rat somatosensory system. J Neurosci 19: 7603-7616, 1999.

Fanselow EE, SameshimaK, Baccala LA, and Nicolelis MA. Thalamic bursting in rats during different awake behavioral states. Proc Natl Acad Sci USA 15330-15335, 2001.

Friedberg MH, Lee SM, and Ebner FF. Modulation of receptive field properties of thalamic somatosensory neurons by the depth of anesthesia. J Neurophysiol 81: 2243-2252, 1999.

Gottschaldt K-M, Vahle-Hinz C, and Hicks TP. Electrophysiological and micropharmacological studies on mechanisms of input-output transformation in single neurones of the somatosensory thalamus. In: Somatosensory Integration in the Thalamus, edited by Macchi G, Rustioni A, and Spreafico R. Amsterdam: Elsevier Science Publishers, 1983, p. 199-216.

Hamos JE, Van Horn SC, Raczkowski D, and Sherman SM. Synaptic circuits involving an individual retinogeniculate axon in the cat. J Comp Neurol 259: 165-192, 1987.

Haring JH, Henderson TA, and Jacquin MF. Principalis- or parabrachialprojecting spinal trigeminal neurons do not stain for GABA or GAD. Somatosens Mot Res 7: 391-397, 1990.

Harris RM. Morphology of physiologically identified thalamocortical relay neurons in the rat ventrobasal thalamus. J Comp Neurol 251: 491-505, 1986.

Harris RM and Hendrickson AE. Local circuit neurons in the rat ventrobasal thalamus: a GABA immunocytochemical study. Neuroscience 21: 229236, 1987.

Hartings JA and Simons DJ. Thalamic relay of afferent responses to 1- to 12-Hz whisker stimulation in the rat. J Neurophysiol 80: 1016-1019, 1998.

Hartings JA and Simons DJ. Inhibition suppresses transmission of tonic vibrissa-evoked activity in the rat ventrobasal thalamus. J Neurosci 20: RC100, 2000.

Hartings JA, Temereanca S, and Simons DJ. High responsiveness and direction sensitivity of neurons in the rat thalamic reticular nucleus to vibrissal deflections. J Neurophysiol 83: 2791-2801, 2000.

Humphrey AL and Weller RE. Functionally distinct groups of X-cells in the lateral geniculate nucleus of the cat. J Comp Neurol 268: 429-447, 1988.

Jacquin MF, Barcia M, and Rhoades RW. Structure- function relationships in rat brainstem subnucleus interpolaris. IV. Projection neurons. J Comp Neurol 282: 45-62, 1989.

Jacquin MF, Chiaia NL, Haring JH, and Rhoades RW. Intersubnuclear connections within the rat trigeminal brainstem complex. Somatosens Mot Res 7: 399-420, 1990.

Jacquin MF, Mooney RD, and Rhoades RW. Morphology, response properties, and collateral projections of trigeminothalamic neurons in brainstem subnucleus interpolaris of rat. Exp Brain Res 61: 457-468, 1986.

Kelly MK, Carvell GE, Hartings JA, and Simons DJ. Axonal conduction properties of antidromically identified neurons in rat barrel cortex. Somatosens Mot Res 18: 202-210, 2001.

Kelly MK, Carvell GE, Jobling J, and Simons DJ. Sensory loss by selected whisker removal produces immediate disinhibition in the somatosensory cortex of behaving rats. $J$ Neurosci 19: 9117-9125, 1999.

Kuffler SW. Discharge patterns and functional organization of mammalian retina. J Neurophysiol 16: 37-68, 1953.

Kyriazi HT, Carvell GE, and Simons DJ. OFF response transformations in the whisker/barrel system. J Neurophysiol 72: 392-401, 1994.

Kyriazi HT and Simons DJ. Thalamocortical response transformations in simulated whisker barrels. J Neurosci 13: 1601-1615, 1993.

Landry $\mathbf{P}$ and Dykes RW. Identification of two populations of corticothalamic neurons in cat primary somatosensory cortex. Exp Brain Res 60: 289298, 1985.

Lee SM, Friedberg MH, and Ebner FF. The role of GABA-mediated inhibition in the rat ventral posterior medial thalamus. I. Assessment of receptive field changes following thalamic reticular nucleus lesions. $\mathrm{J} \mathrm{Neu-}$ rophysiol 71: 1702-1715, 1994a.

Lee SM, Friedberg MH, and Ebner FF. The role of GABA-mediated inhibition in the rat ventral posterior medial thalamus. II. Differential effects of $\mathrm{GABA}_{\mathrm{A}}$ and $\mathrm{GABA}_{\mathrm{B}}$ receptor antagonists on responses of VPM neurons. J Neurophysiol 71: 1716-1726, 1994b.

Lichtenstein SH, Carvell GE, and Simons DJ. Responses of rat trigeminal ganglion neurons to movements of vibrissae in different directions. Somatosens Mot Res 7: 47-65, 1990.

Ma PM. The barrelettes-architectonic vibrissal representations in the brainstem trigeminal complex of the mouse. I. Normal structural organization. J Comp Neurol 309: 161-199, 1991. 
Mastronarde DN. Two classes of single-input X-cells in cat lateral geniculate nucleus. II. Retinal inputs and the generation of receptive-field properties. J Neurophysiol 57: 381-413, 1987.

Mastronarde DN. Nonlagged relay cells and interneurons in the cat lateral geniculate nucleus: receptive-field properties and retinal inputs. Vis Neurosci 8: 407-441, 1992.

Minnery BS and Simons DJ. Response properties of whisker-associated trigeminothalamic neurons in rat nucleus principalis. J Neurophysiol 89: 40-56, 2003.

Nicolelis MA and Fanselow EE. Thalamocortical optimization of tactile processing according to behavioral state. Nat Neurosci 5: 517-523, 2002.

Ohara PT and Havton LA. Dendritic architecture of rat somatosensory thalamocortical projection neurons. J Comp Neurol 341: 159-171, 1994.

Paxinos G and Watson C. The Rat Brain in Stereotaxic Coordinates. San Diego, CA: Academic Press, 1998

Pellegrino LJ, Pellegrino AS, and Cushman AJ. A Stereotaxic Atlas of the Rat Brain. New York: Plenum Press, 1979.

Peschanski M. Trigeminal afferents to the diencephalon in the rat. Neuroscience 12: 465-487, 1984

Pierret T, Lavallee P, and Deschênes M. Parallel streams for the relay of vibrissal information through thalamic barreloids. J Neurosci 20: 74557462, 2000.

Pinault $\mathbf{D}$ and Deschênes M. Anatomical evidence for a mechanism of lateral inhibition in the rat thalamus. Eur J Neurosci 10: 3462-3469, 1998a.

Pinault $D$ and Deschênes M. Projection and innervation patterns of individual thalamic reticular axons in the thalamus of the adult rat: a three-dimensional, graphic, and morphometric analysis. J Comp Neurol 391: 180-203, 1998b.

Pinto DJ, Brumberg JC, and Simons DJ. Circuit dynamics and coding strategies in rodent somatosensory cortex. J Neurophysiol 83: 1158-1166, 2000 .

Pinto DJ, Brumberg JC, Simons DJ, and Ermentrout GB. A quantitative population model of whisker barrels: re-examining the Wilson- Cowan equations. J Comput Neurosci 3: 247-264, 1996.

Pinto DJ, Hartings JA, Brumberg JC, and Simons DJ. Cortical damping: analysis of thalamocortical response transformations in rodent barrel cortex. Cereb Cortex 13: 33-44, 2002

Rhoades RW, Belford GR, and Killackey HP. Receptive-field properties of rat ventral posterior medial neurons before and after selective kainic acic lesions of the trigeminal brain stem complex. J Neurophysiol 57: 1577$1600,1987$.

Sherman SM. Tonic and burst firing: dual modes of thalamocortical relay. Trends Neurosci 24: 122-126, 2001.

Shipley MT. Response characteristics of single units in the rat's trigeminal nuclei to vibrissa displacements. J Neurophysiol 37: 73-90, 1974.

Shosaku A. A comparison of receptive field properties of vibrissa neurons between the rat thalamic reticular and ventro-basal nuclei. Brain Res 347 36-40, 1985

Simons DJ. Multi-whisker stimulation and its effects on vibrissa units in rat SmI barrel cortex. Brain Res 276: 178-182, 1983.
Simons DJ. Rodent whisker barrels: windows into cerebral cortical function. News Physiol Sci 12: 268-273, 1997.

Simons DJ and Carvell GE. Thalamocortical response transformation in the rat vibrissa/barrel system. J Neurophysiol 61: 311-330, 1989.

Simpson KL, Altman DW, Wang L, Kirifides ML, Lin RC, and Waterhouse BD. Lateralization and functional organization of the locus coeruleus projection to the trigeminal somatosensory pathway in rat. J Comp Neurol 385: 135-147, 1997.

Sosnik R, Haidarliu S, and Ahissar E. Temporal frequency of whisker movement. I. Representations in brain stem and thalamus. J Neurophysiol 86: 339-353, 2001

Sumitomo I and Iwama K. Neuronal organization of rat thalamus for processing information of vibrissal movements. Brain Res 415: 389-392, 1987.

Swadlow HA. Efferent neurons and suspected interneurons in S-1 vibrissa cortex of the awake rabbit: receptive fields and axonal properties. J Neurophysiol 62: 288-308, 1989.

Swadlow HA and Hicks TP. Somatosensory cortical efferent neurons of the awake rabbit: latencies to activation via supra- and subthreshold receptive fields. J Neurophysiol 75: 1753-1759, 1996.

Temereanca $\mathbf{S}$ and Simons DJ. Topographic specificity in the functional effects of corticofugal feedback in the whisker/barrel system. Soc Neurosci Abstr 27: 393.6, 2001.

Usrey WM, Reppas JB, and Reid RC. Specificity and strength of retinogeniculate connections. J Neurophysiol 82: 3527-3540, 1999.

Vahle-Hinz C and Gottschaldt KM. Principal differences in the organization of the thalamic face representation in rodents and felids. In: Somatosensory Integration in the Thalamus, edited by Macchi G, Rustioni A, and Spreafico R. Amsterdam: Elsevier Science Publishers, 1983, p. 125-145.

Van der Loos H. Barreloids in mouse somatosensory thalamus. Neurosci Lett 2: 1-6, 1976.

Varga C, Sik A, Lavallee P, and Deschênes M. Dendroarchitecture of relay cells in thalamic barreloids: a substrate for cross-whisker modulation. $\mathrm{J} \mathrm{Neu}-$ rosci 22: 6186-6194, 2002.

Veinante $\mathbf{P}$ and Deschênes $\mathbf{M}$. Single- and multi-whisker channels in the ascending projections from the principal trigeminal nucleus in the rat. J Neurosci 19: 5085-5095, 1999.

Veinante P, Jacquin MF, and Deschênes M. Thalamic projections from the whisker-sensitive regions of the spinal trigeminal complex in the rat. J Comp Neurol 420: 233-243, 2000.

Waite PME. Somatotopic organization of vibrissal responses in the ventrobasal complex of the rat thalamus. J Physiol 228: 527-540, 1973.

Williams MN, Zahm DS, and Jacquin MF. Differential foci and synaptic organization of the principal and spinal projections to the thalamus in rats. Eur J Neurosci 6: 429-453, 1994.

Wilson JR. Synaptic organization of individual neurons in the macaque lateral geniculate nucleus. J Neurosci 9: 2931-2953, 1989.

Woolsey TA and Van der Loos $\mathbf{H}$. The structural organization of layer IV in the somatosensory region (SI) of mouse cerebral cortex. Brain Res 17: 205242, 1970. 